\title{
Phox2b-Expressing Neurons of the Parafacial Region Regulate Breathing Rate, Inspiration, and Expiration in Conscious Rats
}

\author{
Stephen B. G. Abbott, Ruth L. Stornetta, Melissa B. Coates, and Patrice G. Guyenet \\ Department of Pharmacology, University of Virginia, Charlottesville, Virginia 22908
}

The retrotrapezoid nucleus contains Phox2b-expressing glutamatergic neurons (RTN-Phox2b neurons) that regulate breathing in a $\mathrm{CO}_{2}$-dependent manner. Here we use channelrhodopsin-based optogenetics to explore how these neurons control breathing in conscious and anesthetized adult rats. Respiratory entrainment (pacing) of breathing frequency (fR) was produced over $57 \%$ (anesthetized) and $28 \%$ (conscious) of the natural frequency range by burst activation of RTN-Phox $2 \mathrm{~b}$ neurons $(3-8 \times 0.5-20 \mathrm{~ms}$ pulses at $20 \mathrm{~Hz})$. In conscious rats, pacing under normocapnic conditions increased tidal volume $\left(\mathrm{V}_{\mathrm{T}}\right)$ and each inspiration was preceded by active expiration, denoting abdominal muscle contraction. During long-term pacing $\mathrm{V}_{\mathrm{T}}$ returned to prestimulation levels, suggesting that central chemoreceptors such as RTN-Phox $2 b$ neurons regulate $V_{T}$ partly independently of their effect on fR. Randomly applied light trains reset the respiratory rhythm and shortened the expiratory phase when the stimulus coincided with late-inspiration or early-expiration. Importantly, continuous $(20 \mathrm{~Hz})$ photostimulation of the RTN-Phox $2 \mathrm{~b}$ neurons and a saturating $\mathrm{CO}_{2}$ concentration produced similar effects on breathing that were much larger than those elicited by phasic RTN stimulation.

In sum, consistent with their anatomical projections, RTN-Phox2b neurons regulate lung ventilation by controlling breathing frequency, inspiration, and active expiration. Adult RTN-Phox $2 b$ neurons can entrain the respiratory rhythm if their discharge is artificially synchronized, but continuous activation of these neurons is much more effective at increasing lung ventilation. These results suggest that RTN-Phox $2 b$ neurons are no longer rhythmogenic in adulthood and that their average discharge rate may be far more important than their discharge pattern in driving lung ventilation.

\section{Introduction}

The parafacial region of the medulla oblongata regulates breathing (Smith et al., 1989; Janczewski and Feldman, 2006; Guyenet et al., 2010; Kanbar et al., 2010; Marina et al., 2010). This heterogeneous region includes the retrotrapezoid nucleus (RTN), a group of glutamatergic, noncatecholaminergic Phox $2 \mathrm{~b}$-expressing neurons (RTN-Phox $2 \mathrm{~b}$ neurons) that contributes to central respiratory chemoreception (Mulkey et al., 2004; Stornetta et al., 2006; Takakura et al., 2008; Gourine et al., 2010; Guyenet et al., 2010; Marina et al., 2010).

During the perinatal period, RTN-Phox $2 \mathrm{~b}$ neurons are both "inspiratory rhythmogenic" and central respiratory chemoreceptors, i.e., they are $\mathrm{pH}$ sensitive, have intrinsic bursting properties, and their synchronized discharge defines the breathing rate by pacing the pre-Bötzinger complex (Onimaru et al., 2008; ThobyBrisson et al., 2009; Goridis and Brunet, 2010). In adulthood, the respiratory rhythm seems generated by a more distributed net-

Received June 28, 2011; revised Sept. 2, 2011; accepted Sept. 21, 2011.

Author contributions: S.B.G.A., R.L.S., and P.G.G. designed research; S.B.G.A., R.L.S., M.C., and P.G.G. performed research; S.B.G.A., R.L.S., and P.G.G. analyzed data; S.B.G.A., R.L.S., and P.G.G. wrote the paper.

This work was supported by grants from the National Institutes of Health to P.G.G. (HL74011 and HL28785). S.B.G.A. is supported by a postdoctoral fellowship from the American Heart Association (11POST7170001).

Correspondence should be addressed to Dr. Patrice G. Guyenet, University of Virginia Health System, P.O. Box 800735, 1300 Jefferson Park Avenue, Charlottesville, VA 22908-0735. E-mail: pgg@virginia.edu.

DOI:10.1523/JNEUROSCI.3280-11.2011

Copyright $\odot 2011$ the authors $\quad 0270-6474 / 11 / 3116410-13 \$ 15.00 / 0$ work of pontomedullary neurons although the pre-Bötzinger complex remains the network's lynchpin (Rubin et al., 2009; Nattie and Li, 2009; Abdala et al., 2009b; Feldman, 2011). While RTN-Phox $2 b$ neurons retain their central chemoreceptor properties and their ability to activate the respiratory rhythm in adulthood (Li and Nattie, 2002; Mulkey et al., 2004; Takakura et al., 2008; Gourine et al., 2010; Guyenet et al., 2010; Marina et al., 2010), the persistence of their rhythmogenic properties remains controversial (Guyenet et al., 2005; Oku et al., 2007).

Activation of RTN-Phox2b neurons increases breathing rate and inspiratory effort dramatically in conscious and anesthetized adult rats (Abbott et al., 2009a; Kanbar et al., 2010). In other preparations, inhibiting these neurons primarily impacts active expiration (Abdala et al., 2009a), with modest effects on rate and inspiratory effort (Marina et al., 2010). According to others, active expiration is generated by a parafacial expiratory oscillator that does not include the RTN-Phox2b neurons (Janczewski and Feldman, 2006; Pagliardini et al., 2011).

We postulate that, in the intact adult brain, the RTN-Phox $2 \mathrm{~b}$ neurons recruit inspiratory and expiratory muscles in an orderly sequence that depends on the degree to which they are activated by $\mathrm{CO}_{2}$ or by other mechanisms (Guyenet et al., 2010). This notion stems primarily from three observations. Anatomically verified RTN-Phox $2 \mathrm{~b}$ neurons are activated by $\mathrm{CO}_{2}$, both in slices and in vivo (Guyenet et al., 2005; Lazarenko et al., 2009). Selective activation of RTN-Phox2b neurons powerfully in- 
creases breathing rate and tidal volume in conscious rats (Kanbar et al., 2010). RTN-Phox 2 b neurons innervate all segments of the pontomedullary respiratory network, including where bulbospinal expiratory premotor neurons reside (Abbott et al., 2009a; Bochorishvili et al., 2011).

In the present study we used channelrhodopsin-based optogenetics to test whether stimulation of RTN-Phox $2 b$ neurons in conscious rats activates the three major components of breathing (rate, inspiration, and active expiration) and whether the respiratory frequency can be set by phasically activating these neurons.

\section{Materials and Methods}

Animals. The experiments were performed in male Sprague Dawley rats (Taconic) in accordance with NIH and Institutional Animal Care and Use Guidelines. The Animal Research Committee of the University of Virginia approved all procedures and protocols.

Lentiviral vector and injection. We used a previously described lentiviral vector that expresses enhanced humanized channelrhodopsin-2 fused with mCherry under the control of the artificial Phox2b-specific promoter PRSx8 [pLenti-PRSx8-hChR2 (H134R)-mCherry-WPRE, henceforth abbreviated PRSx8-ChR2-mCherry] (Hwang et al., 2001; Abbott et al., 2009b). Phox $2 b$ is a transcription factor expressed in subsets of brainstem neurons, including the RTN and catecholaminergic cells (Pattyn et al., 1997; Stornetta et al., 2006). The virus was produced by the University of North Carolina Virus Core and diluted to a final titer of $3.0 \times 10^{8}$ viral particles $\cdot \mathrm{ml}^{-1}$ with sterile PBS before injection into brain. We verified that the above viral dilution produced specific transfection of Phox $2 b-$ expressing neurons (see Results).

Surgery for lentiviral injection was performed in 225-250 g rats under anesthesia [ketamine $(75 \mathrm{mg} / \mathrm{kg})$, xylazine $(5 \mathrm{mg} / \mathrm{kg})$, and acepromazine $(1 \mathrm{mg} / \mathrm{kg})$, administered i.m.] using standard aseptic methods. A glass pipette with external tip diameter of $25 \mu \mathrm{m}$ was inserted into the brain through a dorsal craniotomy. The lentivirus was delivered through the pipette by controlled pressure injection (60 psi, 3-8 ms pulses). The injections were targeted unilaterally to four sites separated by $200 \mu \mathrm{m}$ $(2 \times 2$ grid; total volume, $420 \mathrm{nl})$. These sites were located $100-200 \mu \mathrm{m}$ below the caudal end of the facial motor nucleus, whose lower edge was identified in each rat by antidromic mapping as described previously (Abbott et al., 2009b). In two animals, anti-dopamine- $\beta$-hydroxylase antibody conjugated to saporin (antiD $\beta \mathrm{H}$-sap; Advanced Targeting Systems) was also injected at $0.22 \mu \mathrm{g} / \mu \mathrm{l}$ bilaterally (two sites total, $100 \mathrm{nl}$ per site) into the region of the lateral horn of the second thoracic segment (1.0-1.2 mm lateral of midline, $1 \mathrm{~mm}$ below the lateral sulcus) to destroy the $\mathrm{C} 1$ neurons that project to the spinal cord. For experiments in conscious rats, a guide cannula (Plastics One) was implanted 1 week after virus injection at the same stereotaxic coordinates as those of the injections of virus. This cannula extended $5 \mathrm{~mm}$ below the surface of the skull and was secured with jeweler screws and dental cement. In all animals, the craniotomy was patched with sterile absorbable cellulose and wounds were closed with sutures, wound clips, and/or cyanoacrylic glue. Following surgery, rats were treated at 0 and $24 \mathrm{~h}$ with an antibiotic (ampicillin, $100 \mathrm{mg} / \mathrm{kg}$, i.m.) and analgesic (ketorolac, $0.6 \mathrm{mg} / \mathrm{kg}$, i.p.). Animals were maintained for no less than 2 weeks before they were used in physiological experiments. The surgical procedures, virus injections, and guide cannula placement produced no observable behavioral or respiratory effects, and these rats gained weight normally.

Physiological experiments in anesthetized rats $(350-425 \mathrm{~g} ; \mathrm{N}=7)$. A tracheostomy was performed under general anesthesia with $5 \%$ isoflurane in $100 \%$ oxygen. Artificial ventilation $(1 \mathrm{ml} / 100 \mathrm{~g}, 60-80$ cycles per $\mathrm{min}$ ) was initiated with $3.0-3.5 \%$ isoflurane in pure oxygen and maintained throughout surgical procedures. Frequency of ventilation was adjusted as needed to maintain end-tidal $\mathrm{CO}_{2}\left(\mathrm{etCO}_{2}\right)$ at the desired level. Blood gases were not measured. Arterial $\mathrm{pCO}_{2}$ (partial pressure of carbon dioxide) was estimated from measurements of end-tidal $\mathrm{CO}_{2}$ (Guyenet et al., 2005). This variable was monitored with a microcapnometer (Columbus Instruments). Positive end-expiratory pressure $\left(1.5 \mathrm{~cm} \mathrm{H}_{2} \mathrm{O}\right.$ ) was maintained throughout to minimize atelectasis. Mean blood pressure remained above $110 \mathrm{mmHg}$, and the phrenic nerve activ- ity could be silenced by lowering end-tidal $\mathrm{pCO}_{2}$ to $3.5-4.5 \%$. In all animals, the femoral artery and vein were cannulated, and both vagus nerves were cut distal to the carotid bifurcation as described previously (Guyenet et al., 2005). The right phrenic nerve was dissected and recorded to monitor central respiratory drive to the diaphragm (Moreira et al., 2006; Takakura et al., 2006). The light stimulus was delivered with a $200 \mu \mathrm{m}$ diameter fiber optic (Thorlabs) inserted vertically into the brainstem. To increase the accuracy of the fiber optic placement, the facial motor nucleus was mapped with antidromic field potentials using a glass pipette and, based on these coordinates, the optical fiber was positioned $\sim 200-400 \mu \mathrm{m}$ dorsal to the retrotrapezoid nucleus.

Upon completion of surgical procedures, urethane $(1.3 \mathrm{~g} / \mathrm{kg}, 20 \% \mathrm{w} / \mathrm{v}$ in sterile $\mathrm{H}_{2} \mathrm{O}$ ) was slowly infused intravenously and isoflurane was withdrawn. Supplementary doses of $0.1 \mathrm{~g} / \mathrm{kg}$ urethane were administered intravenously as needed (up to an additional $0.25 \mathrm{~g} / \mathrm{kg}$ during the course of an experiment) to maintain an appropriate depth of anesthesia based on the absence of respiratory changes and blood pressure changes of $<10$ $\mathrm{mmHg}$ in response to a firm paw pinch. A minimum of $30 \mathrm{~min}$ was allowed following the removal of isoflurane before commencing experiments. At that point, rats were administered the muscle relaxant pancuronium (initial dose, $1 \mathrm{mg} / \mathrm{kg}$ i.v. plus additional $0.2 \mathrm{mg} / \mathrm{kg}$ doses if needed).

Integrated phrenic nerve discharge (iPND) was obtained after rectification and smoothing ( $\tau=0.03 \mathrm{~s}$ ) of the original signal (bipolar recording, $0.2-3 \mathrm{kHz}$ band). The product of the respiratory frequency (fR, in $\mathrm{min}^{-1}$ ) times the amplitude (amp) of iPND (PNDamp, in arbitrary units) was used to measure the total respiratory neural output of the preparation per minute $(\mathrm{fR} \times \mathrm{amp})$. PNDamp and $\mathrm{fR} \times$ amp were normalized by assigning a value of 100 to the maximum amplitude of iPND or $\mathrm{fR} \times$ amp observed at saturation of the chemoreflex $(\sim 8 \%$ et $\mathrm{CO}_{2}$ ) within each experiment and a value of 0 to periods of apnea.

Whole-body unrestrained plethysmography in conscious rats (300-400 $g ; \mathrm{N}=6)$. Tidal volume $\left(\mathrm{V}_{\mathrm{T}}, \mathrm{ml}\right)$, breathing frequency ( $\mathrm{fR}$, breaths $\left.\mathrm{min}^{-1}\right)$, and minute volume $\left(\mathrm{MV}, \mathrm{ml} \cdot \mathrm{min}^{-1}\right)$ were evaluated by wholebody plethysmography. The rats were placed in a Perspex flow-through chamber (Buxco) and allowed $1-2 \mathrm{~h}$ to acclimate to their new surroundings. Afterward, rats were briefly ( $<5 \mathrm{~min})$ anesthetized with isoflurane $\left(1.5 \%\right.$ maintenance after $3 \%$ induction in $\mathrm{O}_{2}$ ) to allow insertion of a custom optic fiber assembly into the implanted guide. A fiber length of $9.5-10 \mathrm{~mm}$ produced the best results. Animals were returned to the plethysmography chamber and allowed to recover from anesthesia over 30 min before commencing experimental protocols. The chamber was continuously flushed with dry room temperature air $\left(24 \pm 0.5^{\circ} \mathrm{C}\right)$ delivered by three computer-driven mass flow regulators connected to pure $\mathrm{O}_{2}, \mathrm{~N}_{2}$, and $\mathrm{CO}_{2}\left(1 \mathrm{~L} \mathrm{~min}^{-1}\right)$. The flow signal was recorded and analyzed using EMKA IOX 2.7 (EMKA Technologies) and Spike 5.21 (CED) software. Breathing parameters were calculated from a calibrated flow signal derived from a differential pressure sensor connected to the plethysmography chamber using the equation of Drorbaugh and Fenn (1955). Realtime chamber conditions (temperature and humidity) and atmospheric pressure were continuously measured, and the calculation of tidal volume was automatically adjusted to account for changes in these variables.

Photostimulation parameters. Photostimulation of the ventrolateral medulla was done with a diode-pumped $473 \mathrm{~nm}$ blue laser (CrystaLaser) controlled by a Grass model S88 stimulator (AstroMed). The light output of the laser at the tip of the optical fiber was measured before implantation into the brain and was always set at $9 \mathrm{~mW}$ as per our prior work (Abbott et al., 2009a). The respiratory effects elicited by continuous stimulation at $20 \mathrm{~Hz}$ were a saturable function of the pulse width. RTN neurons fire up to $20 \mathrm{~Hz}$ under anesthesia (Fortuna et al., 2009). Their dynamic range in a conscious animal is unknown. A frequency of 20-23 $\mathrm{Hz}$ was chosen based on prior evidence that using the same $\mathrm{CHR} 2$ variant (H134R), these neurons can follow this frequency in anesthetized rats (Abbott et al., 2009a). To minimize potential heat damage to the tissue, we determined the shortest pulse required to produce the maximum ventilatory effect in each rat and used this parameter throughout the experiment (range, $0.5-10 \mathrm{~ms}$ ). Train photostimulation was done with brief stimulus trains (anesthetized rats: $5-20 \mathrm{~ms}$ light pulses, $20 \mathrm{~Hz}$, train duration of $328 \pm 18 \mathrm{~ms}$; conscious rats: $1-10 \mathrm{~ms}$ pulses, $20-23 \mathrm{~Hz}$, train 
duration of $200 \pm 48 \mathrm{~ms}$ ). Each train lasted for the equivalent of $59 \pm 5^{\circ}$ (anesthesia) and $91 \pm 24^{\circ}$ (conscious) of the total respiratory cycle.

Data analysis and statistics. Analysis was done using Excel 2010 and GraphPad 10 software. Results are presented as mean \pm SEM. Student's $t$ test and one- and two-way ANOVA were used as required.

Periods of respiratory pacing were identified by the constant latency between the onset of light trains and inspiration, and a 1:1 coupling of the light train and inspiration for $>15$ cycles. For the description of the range of entrainment and the average stimulation phase during entrainment, $15-20$ cycles of entrainment at each stimulation frequency were extracted and averaged for each animal and expressed in $\mathrm{Hz}$ before being grouped. The stimulus trains used to test for respiratory entrainment were delivered in step increments of 5 bursts $\cdot \min ^{-1}(0.83 \mathrm{~Hz})$. Train frequency ranged from resting frequency to the maximum breathing rate observed during hypercapnia, although every increment was not tested in each animal. In some cases each stimulation bout was separated by a rest period, and in others cases the frequency was stepped up and down in a staircase fashion and maintained at the various plateaus for around 1 min. The "stimulus phase" represents the interval between the beginning of inspiration and the onset of the stimulus and is expressed as a fraction of the total respiratory cycle $\left(360^{\circ}\right)$.

The effects of artificially increasing $\mathrm{fR}$ on $\mathrm{V}_{\mathrm{T}}$ and $\mathrm{MV}$ in conscious rats was assessed by calculating the average of the first and last 10 breaths (early and late, respectively) of an entrainment period and comparing these values to a period of unperturbed breathing (rest). Data for this analysis was taken from periods when $\mathrm{fR}$ was held at a single elevated entrainment frequency or over several increments in entrainment frequency.

To evaluate active expiration, we measured the average expiratory volume (15-20 breaths) during the first and the second half of the expiratory period and measured the ratio between the two (late: early expiration).

Phase resetting was evaluated by comparing the induced phase (time from the onset of the stimulus to inspiration in next respiratory cycle) against the stimulus phase. In this analysis, the stimulus phase reflects the period from inspiration to the stimulus in relation to the period of the preceding unperturbed respiratory cycle (i.e., expected phase or $\mathrm{N}-1$ ). The phase of the $\mathrm{N}+1$ cycle in this analysis was calculated by combining the period of the induced phase and $\mathrm{N}+1$ and dividing this value by the expected phase.

To analyze the perturbations in respiratory timing and effort produced by brief activation of Phox $2 \mathrm{~b}$ neurons, we evaluated the changes in inspiratory time $\left(\mathrm{T}_{\mathrm{I}}\right)$, expiratory time $\left(\mathrm{T}_{\mathrm{E}}\right)$, total respiratory cycle time $\left(\mathrm{T}_{\mathrm{Tot}}\right.$ ), and peak iPND (anesthetized) or $\mathrm{V}_{\mathrm{T}}$ (conscious) produced by trains of light delivered at low frequency randomly within the respiratory cycle. For $\mathrm{T}_{\mathrm{E}}$ and $\mathrm{T}_{\mathrm{Tot}}$, the perturbed respiratory cycle containing the stimulus train $(\mathrm{N})$ and the following respiratory cycle $(\mathrm{N}+1)$ were normalized against the unperturbed cycle preceding $\mathrm{N}$ (i.e., $\mathrm{N}-1$ or control cycle). This analysis controls for long-term changes in respiratory cycle time and effort. All data were grouped according to stimulus phase ( $30^{\circ}$ bands), and significant phase perturbations of the $\mathrm{T}_{\mathrm{E}}$ and $\mathrm{T}_{\text {Tot }}$ were determined by comparing the values of normalized $\mathrm{N}$ versus $\mathrm{N}+1$ cycle using a two-way ANOVA with Bonferroni's multiple comparisons. Analysis of $\mathrm{T}_{\mathrm{I}}$ and peak PND or $\mathrm{V}_{\mathrm{T}}$ required an evaluation of 3 breaths $(\mathrm{N}, \mathrm{N}+1$, and $\mathrm{N}+2$, where $\mathrm{N}+2$ is the breath following $\mathrm{N}+1$ ), because the $\mathrm{N}$ and $\mathrm{N}+1$ cycle were both subject to the effects of the stimulus; for example, a late stimulus phase interacts solely with the $\mathrm{N}+1 \mathrm{~T}_{\mathrm{I}}$ cycle and not the $\mathrm{N}_{\mathrm{I}}$ cycle because the stimulus occurs after the end of the $\mathrm{N} \mathrm{T}_{\mathrm{I}}$ cycle. The $\mathrm{T}_{\mathrm{I}}$ and PNDamp or $\mathrm{V}_{\mathrm{T}}$ of the $\mathrm{N}$ and $\mathrm{N}+1$ (presented separately) were normalized against the $\mathrm{N}-1$ cycle, grouped as per $\mathrm{T}_{\mathrm{E}}$ and $\mathrm{T}_{\text {Tot }}$ and statistically compared to the $\mathrm{N}+2$ cycle to identify significant perturbations.

Histology. After the end of the physiological procedures the rats were deeply anesthetized with 5\% isoflurane and perfused with buffered $4 \%$ paraformaldehyde. After 1-2 d of postfixation, $30 \mu \mathrm{m}$-thick coronal brain sections were cut with a vibrating microtome (Leica). Coronal brain sections were obtained from every rat to locate the tip of the optical fiber and to determine the distribution and phenotype of the ChR2transfected neurons. In experiments testing the specificity of the virus,
Phox $2 \mathrm{~b}$ was detected using a rabbit antibody (provided by J.-F. Brunet, École Normale Supérieure, Paris, France; 1:800 dilution) and visualized using appropriate fluorescent secondaries depending on the requirements of the experiments. To evaluate the proportion of ChR2mCherry-expressing neurons that were Phox $2 b$-positive, we identified mCherry by its natural fluorescence, and Phox $2 \mathrm{~b}$ was detected by immunohistochemistry as described previously (Abbott et al., 2009a). To evaluate the proportion of catecholaminergic or cholinergic neurons that expressed ChR2-mCherry, all markers were detected by immunohistochemistry (mCherry with Living Colors rabbit anti-DsRed rabbit polyclonal antibody, Clontech, dilution of 1:500; tyrosine hydroxylase with mouse anti-TH or sheep anti-TH, 1:1000, both from Millipore; choline acetyltransferase with goat anti-ChAT, dilution of 1:50, Millipore) followed by appropriate fluorescent secondaries. All histological methods and antibody specificity are detailed by Kang et al. (2007), Abbott et al. (2009a), and Stornetta et al. (2009).

All histological material was examined and photographed using a Zeiss Axioimager.Z1 (Carl Zeiss Microimaging) with a Zeiss Axiocam MRc digital camera (basic resolution, $1388 \times 1040$ pixels). For estimation of the number and phenotype of transfected neurons, a one in six series of 30 micron coronal sections that encompassed the transfected brain area (from 7 to 10 sections/rat) was plotted using the Neurolucida computerassisted graphing software (MicroBrightfield) as described previously (Stornetta et al., 2002).

\section{Results}

\section{High-frequency photostimulation of RTN Phox $2 b$ neurons increases breathing in anesthetized and conscious rats}

Consistent with our previous observations (Abbott et al., 2009a; Kanbar et al., 2010), continuous high-frequency stimulation $(20-23 \mathrm{~Hz}, 30 \mathrm{~s})$ produced extremely large increases in breathing rate and amplitude in anesthetized and conscious normocapnic rats (Fig. $1 A, B$ ). In normocapnic (3.5-5.3\% et $\mathrm{CO}_{2}$ ) anesthetized rats $(N=5), 20 \mathrm{~Hz}$ stimulation increased PND frequency by $206 \%$ (from $16 \pm 2$ to $49 \pm 4$ bursts $\cdot \min ^{-1}, p<0.001$ ), PND amplitude by $57 \%$ (from $42 \pm 6$ to $67 \pm 4$ units, 100 units representing maximal amplitude at saturation of the chemoreflex, $p<0.05)$, and the total respiratory drive $(\mathrm{fR} \times \mathrm{amp})$ by $350 \%$ of baseline (from $16 \pm 5$ to $73 \pm 9$ units, 100 units being the maximum value observed at saturation of the chemoreflex, $p<0.001$ ) (Fig. 1A,C). Mean arterial pressure increased modestly in normocapnic and hypercapnic conditions $(7 \pm 1$ and $9 \pm 2 \mathrm{mmHg}$, respectively; $p=0.38$ ). The light-induced increase in breathing frequency had fast on-kinetics and biphasic off-kinetics, the latter consisting of a virtually instantaneous but partial reduction of $\mathrm{fR}$ followed by a slow, approximately exponential decay to baseline (Fig. $1 A$ and Abbott et al., 2009a). The initial fast component was most conspicuous when the magnitude of the frequency response was large.

When the anesthetized rats were exposed to levels of $\mathrm{CO}_{2}$ that saturated the chemoreflex (etCO $\mathrm{C}_{2}>7 \%$ ), photostimulation at $20 \mathrm{~Hz}$ produced no further increase in breathing parameters (fR: $47 \pm 2$ to $50 \pm 2$ bursts $\cdot \min ^{-1}$, NS; PNDamp: $84 \pm 9$ to $91 \pm 8$ units, NS; fR $\times$ PNDamp: $86 \pm 10$ to $99 \pm 7 \%$ units, NS) (Fig. $1 B, C$ ).

In conscious rats $(N=6)$ breathing quietly in hyperoxic conditions $\left(100 \% \mathrm{O}_{2}\right.$, no added $\left.\mathrm{CO}_{2}\right)$, photostimulation at $20-23 \mathrm{~Hz}$ increased breathing frequency by $202 \%$ (from $79 \pm 7$ to $160 \pm 11$ breaths $\cdot \min ^{-1}, p<0.001$ ), $\mathrm{V}_{\mathrm{T}}$ by $65 \%$ (from $1.5 \pm 0.2$ to $2.3 \pm$ $0.2 \mathrm{ml}, p<0.05$ ), and total ventilation, $\mathrm{MV}$, by $310 \%$ (from $117 \pm 26$ to $\left.363 \pm 33 \mathrm{ml} \cdot \min ^{-1}, p<0.01\right)($ Fig. $1 B, D)$. The onset kinetics were similar in conscious and anesthetized rats. The increase in breathing frequency reached its maximum value almost instantaneously and remained at that level throughout the stimulus, whereas the increase in amplitude had biphasic kinetics 
A
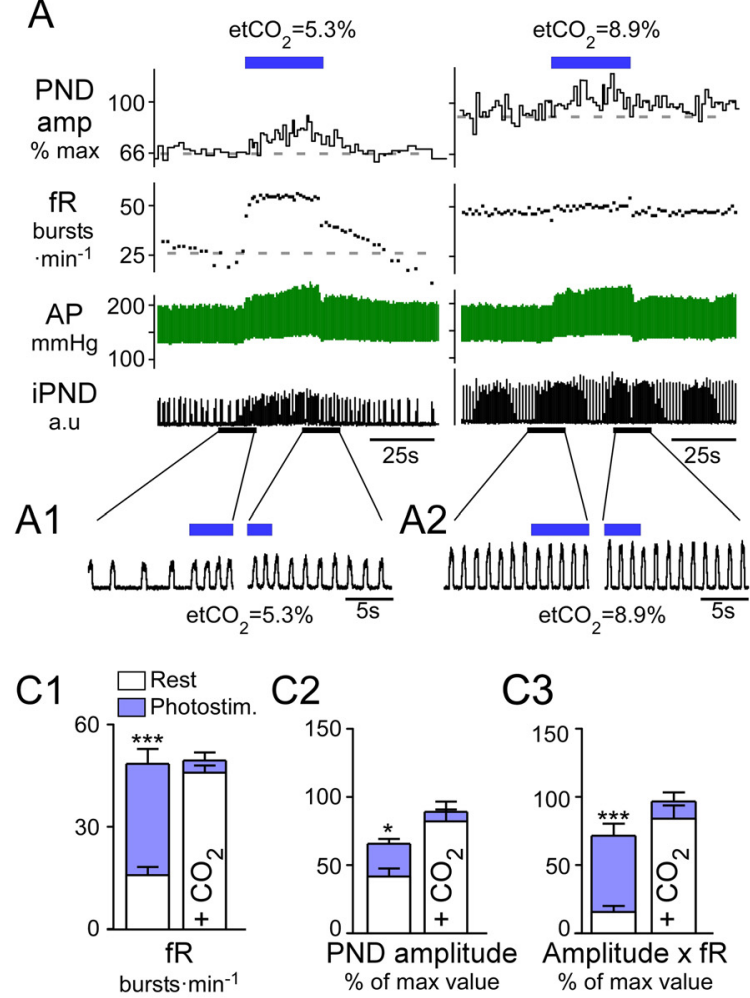

$\mathrm{C} 2$

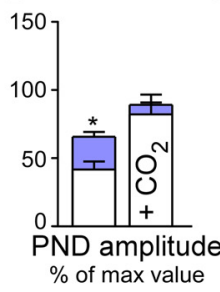

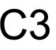

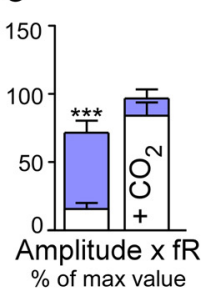

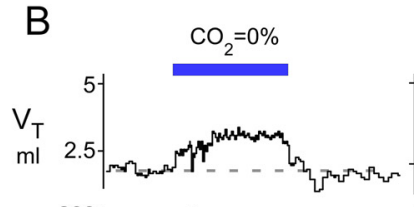
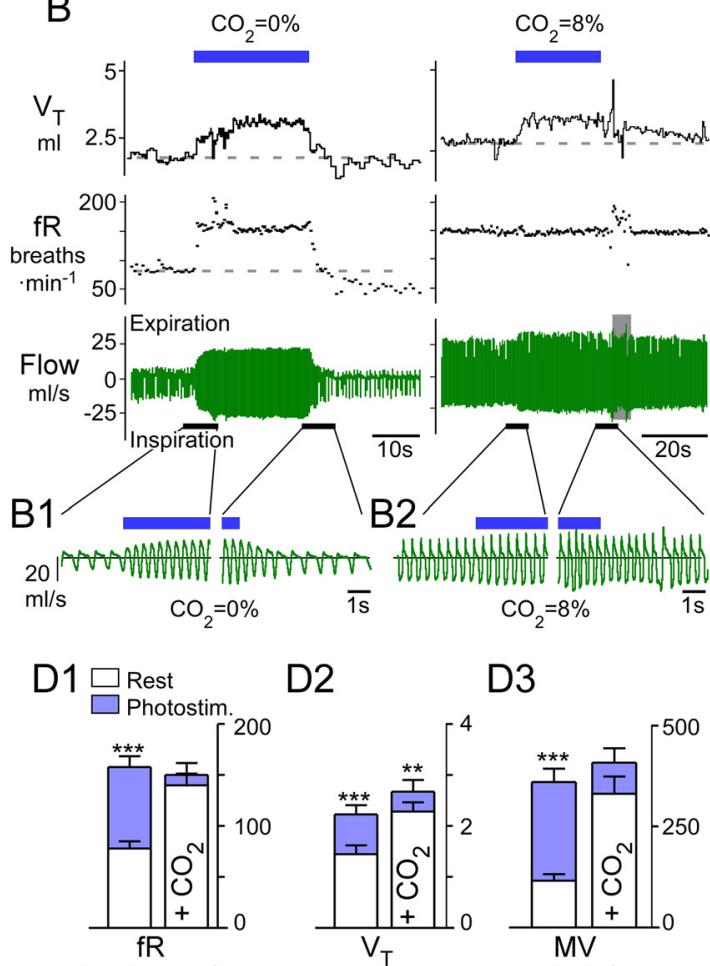

D2

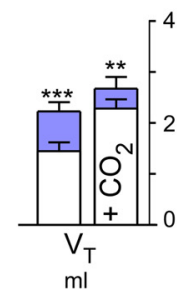

D3

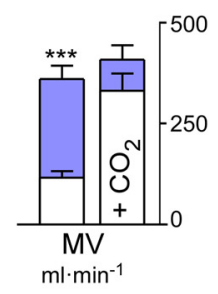

Figure 1. Breathing stimulation elicited by photostimulation of RTN Phox2b neurons is occluded by hypercapnia. $A$, Urethane-anesthetized, vagotomized, and ventilated rat. Left, Normocapnia (etC02,5.3\%). Right, Hypercapnia (etC02, 8.9\%). RTN photostimulation ( $20 \mathrm{~Hz}, 5 \mathrm{~ms}$ pulses) is indicated by the blue bar. Under normocapnia, respiratory frequency, fR, increased within seconds to a steady-state level $>50$ bursts $\cdot \mathrm{min}^{-1}$ and decayed in a two-step fashion (rapid, then slow) upon cessation of the stimulus. Phrenic nerve discharge peak amplitude, PNDamp, progressively increased during the stimulus and also had a slow decay upon cessation of the stimulus. During hyperoxic hypercapnia (right) the same stimulus produced no further increase of fR, a lesser increase in peak amplitude, but an identical increase in arterial pressure (AP). $B$, Conscious rat in flow-through, whole-body plethysmography chamber. Left, Hyperoxic normocapnia ( $100 \% 0_{2}$ ); right, hypercapnia $\left(8 \% \mathrm{CO}_{2}\right.$, balance $\left.\mathrm{O}_{2}\right)$. Continuous RTN stimulation $\left(23 \mathrm{~Hz}, 3\right.$ ms pulses, 30 s total duration, blue bar) raised breathing frequency, $\mathrm{fR}$, and tidal volume, $\mathrm{V}_{\mathrm{T}}$, with identical onset kinetics as that in the anesthetized rat. Following the end of the stimulus, $f R$ and $V_{T}$ dropped precipitously below baseline in the conscious rat, presumably because of hyperventilation-induced hypocapnia. During hyperoxic hypercapnia (right), RTN photostimulation produced a small increase in $V_{T}$ but no increase in $f R . A 1, A 2, B 1, B 2$, Expanded traces highlighting the periods surrounding the beginning and end of the stimulus. Note that in plethysmography records, inspiration is downward. C, Group data for anesthetized rats. From left to right: C1, fR; $\mathbf{C 2}$, PND peak amplitude; and $\mathbf{C 3}$, double product (fR $\times$ PND amplitude) at normal (left columns) and elevated $\mathrm{CO}_{2}$ (right columns). $\boldsymbol{D}$, Group data for conscious rats. From left to right: $\mathbf{D 1}$, fR; $\mathbf{D 2}, \mathrm{V}_{\mathrm{T}} ;$ and $\mathbf{D} \mathbf{3}$, double product $\mathrm{MV}$, minute volume, at normal (left columns) and elevated $\mathrm{CO}_{2}$ (right, $8 \% \mathrm{CO}_{2}$ in pure oxygen). ${ }^{* * *} p<0.001,{ }^{* *} p<0.01,{ }^{*} p<0.05$ between prestimulus baseline and peak values during the stimulation period; two-way ANOVA with multiple comparisons.

consisting of an initial rapid increase followed by a more gradual recruitment. At the end of the stimulation, a large breathing undershoot $\left(\mathrm{fR}\right.$ and $\left.\mathrm{V}_{\mathrm{T}}\right)$ was observed in the conscious rats. This undershoot plausibly resulted from the reduction in arterial $\mathrm{pCO}_{2}$ caused by the preceding hyperventilation, because it was not observed in the anesthetized rats in which the use of muscle relaxants and artificial ventilation should have prevented any change in blood $\mathrm{pCO}_{2}$ during activation of the respiratory network.

As in the anesthetized rats, the effects produced by highfrequency photostimulation were also largely occluded when the conscious rats were exposed to high levels of $\mathrm{CO}_{2}(8 \%$ in pure oxygen; fR from $142 \pm 12$ to $152 \pm 12$ breaths $\cdot \mathrm{min}^{-1}$, NS; MV from $334 \pm 43$ to $411 \pm 36 \mathrm{ml} \cdot \mathrm{min}^{-1}$, NS) (Fig. $1 D$ ), although $\mathrm{V}_{\mathrm{T}}$ was increased over and above the values produced by increasing inspired $\mathrm{CO}_{2}$ to $8 \%$ (from $2.3 \pm 0.2$ to $2.7 \pm 0.2 \mathrm{ml}, p<0.01$; Fig. 1D2).

\section{Activation of RTN Phox $2 b$ neurons paces breathing in} anesthetized and conscious rats

Prior experiments revealed that, in adult rats, activation of the respiratory network by RTN neuron stimulation decays with a 10 second time constant (Abbott et al., 2009a). This result suggested that RTN neurons operate via a type of synaptic transmission that is far too slow for them to have any kind of respiratory rhythmogenic role in adult rodents in which the respiratory cycle lasts from 0.5 to $1 \mathrm{~s}$. We tested whether phasic activation of the RTN neurons has the capability of entraining the respiratory rhythm in the adult. A negative outcome would have ruled out a rhythmogenic function on grounds of excessively slow synaptic transmission, but this is not what we observed.

We applied short trains of laser light (3-8 pulses per train, 20 $\mathrm{Hz}, 0.5-10 \mathrm{~ms}$ pulses) to the RTN region to test whether the breathing rate could be entrained to the stimuli. Entrainment was successful at train frequencies within the intrinsic operating range of breathing frequency. The elevated breathing frequency could be maintained for extended periods of time (range, $0.5-15.5 \mathrm{~min}$ ) in both anesthetized rats (Fig. 2A) and conscious rats (Fig. 2B).

In the urethane-anesthetized rats $(N=7)$, PND frequency varied from $24 \pm 3$ bursts $\cdot \mathrm{min}^{-1}$ just above the apneic threshold $\left(4-5 \%\right.$ et $\left.\mathrm{CO}_{2}\right)$ to $61 \pm 3$ bursts $\cdot \mathrm{min}^{-1}$ at saturation of the chemoreflex (range, $36 \pm 5$ bursts $\cdot \min ^{-1}$ ), with a frequency of $30 \pm 2$ bursts $\cdot \min ^{-1}$ during typical resting conditions. In these rats, intermittent stimulation entrained PND over a range of $21 \pm 4$ bursts $\cdot \mathrm{min}^{-1}$, equivalent to $57 \pm 11 \%$ of the full operating range. 
A

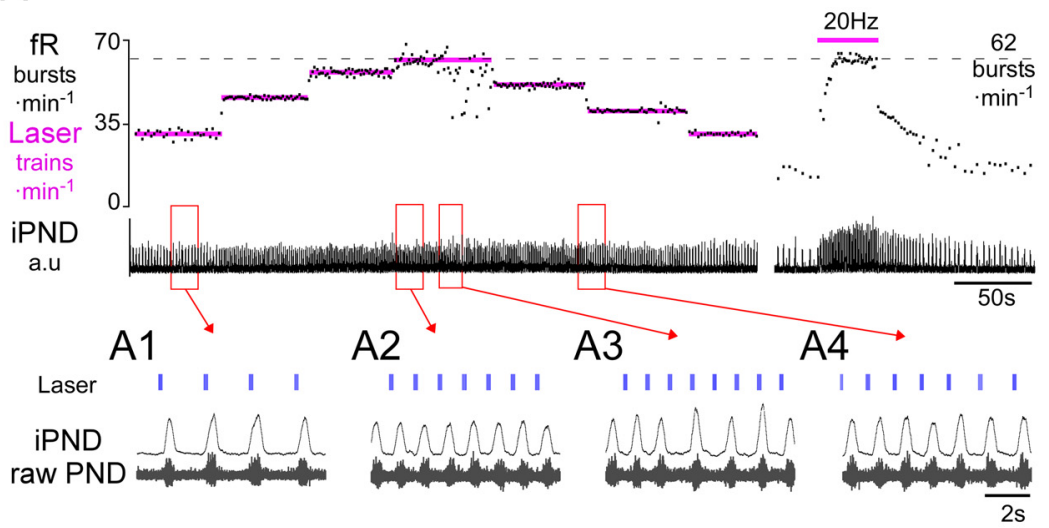

B
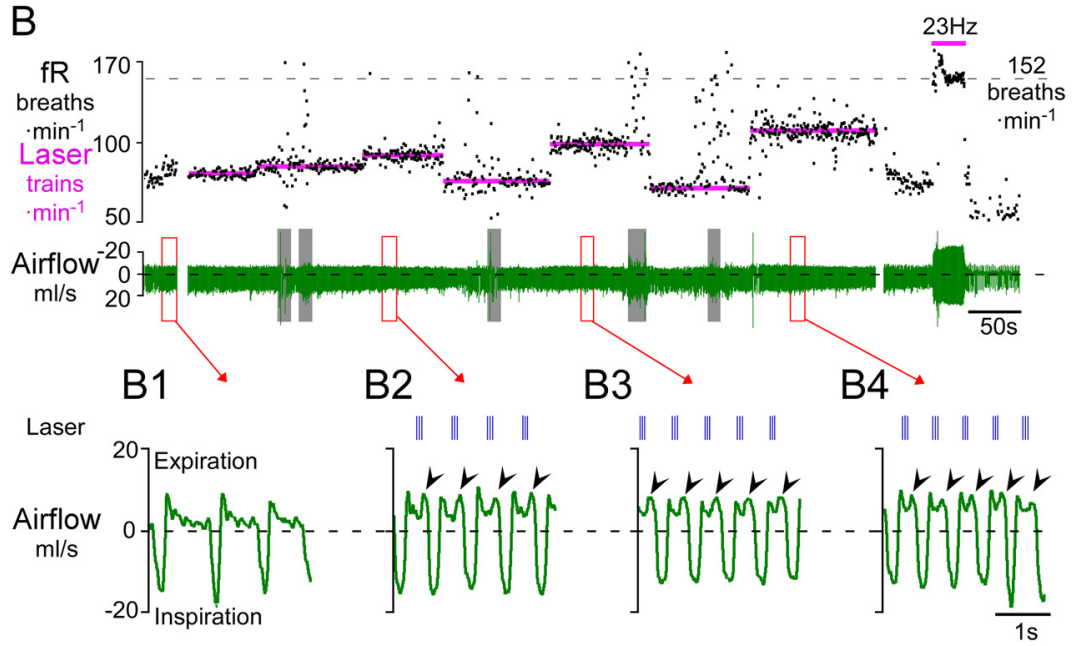

C
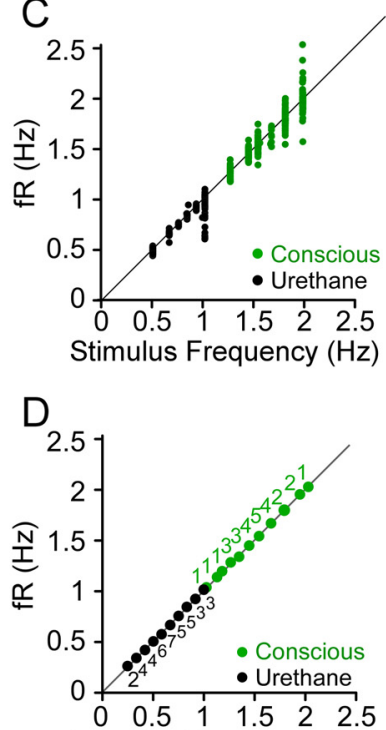

Stimulus Frequency $(\mathrm{Hz})$

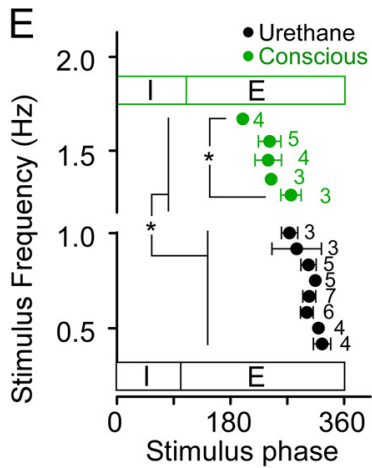

Figure 2. Respiratory pacing by intermittent photostimulation of RTN neurons in anesthetized and conscious rats. $A$, Urethane-anesthetized rat. Photostimulus trains $(4 \times 20 \mathrm{~ms}$ pulses at $20 \mathrm{~Hz})$ entrained PND over a range of 32 breaths $\cdot \min ^{-1}$ (from 25 to 60 bursts $\cdot \min ^{-1}$; see also excerpts $A 1, A 2$ ). The maximal fR observed in this rat during continuous RTN stimulation at $20 \mathrm{~Hz}$ was 62 bursts $\cdot \min ^{-1}$ (at right in $\boldsymbol{A}$ ). At low frequencies ( $\boldsymbol{A} \mathbf{1}$ ) the laser trains settled in late-expiration, whereas at higher frequencies the stimulus train settled earlier during the expiratory phase (A2). RTN stimulation at 60 trains $\cdot \min ^{-1}$ caused arrhythmia (excerpt $\left.A 3\right)$. A4 shows that fR follows the transition from one stimulus frequency to the next $\left(50\right.$ to 40 trains $\cdot$ min $\left.^{-1}\right)$. B, Conscious quiet rat. Light pulses $\left(3 \times 3 \mathrm{~ms}\right.$ pulses at $20 \mathrm{~Hz}$ ) entrained $\mathrm{fR}$ in the range of $75-120$ breaths $\cdot \mathrm{min}^{-1}$. Resting $\mathrm{fR}$ was close to $60 \mathrm{~min}^{-1}$ in this rat, and the maximum $\mathrm{fR} \mathrm{seen} \mathrm{in} \mathrm{presence} \mathrm{of} \mathrm{high} \mathrm{CO}_{2}$ was 152 breaths $\cdot \min ^{-1}$. Continuous high-frequency stimulation $(23 \mathrm{~Hz}, 3 \mathrm{~ms}$, at right in $B$ ) produced a more robust activation of breathing than short bursts. Gray bars indicate periods of behavioral artifacts (e.g., sniffing, movement). $\boldsymbol{B}$ 1-B4 excerpts from $\boldsymbol{B}$ show expanded plethysmography traces at various entrainment frequencies. $\boldsymbol{B} 1$, Quiet breathing in the absence of stimulation. Note monophasic decay of airflow during expiration consistent with passive lung recoil. B2-B4, Airflow traces at three different pacing frequencies. Expiratory flow becomes biphasic with pronounced increase in flow during late-expiration (arrowheads), indicative of active expiration. Note that the stimulation trains settle in mid-expiration during entrainment. $C$, Relationship between stimulus train frequency and fR for the rats shown in $\boldsymbol{A}$ (anesthetized, black) or $\boldsymbol{B}$ (conscious, green). The instantaneous frequency of each burst/breath is plotted against the frequency of the light trains. Note the increased variability of fR at higher entrainment frequencies, reflecting poor entrainment. $\boldsymbol{D}$, Group data showing the reliability of entrainment at a range of stimulus frequencies in anesthetized $(N=7)$ and conscious $(N=6)$ animals. Each point represents the average $f R( \pm S E M)$ when entrainment was successful. Adjacent numbers indicate the number of animals that were successfully entrained at a given stimulation frequency. Not every frequency was tested in every animal; hence, the varying number of determinations for each tested frequency. $\boldsymbol{E}$, Relationship between the stimulus train frequency and the phase angle of the stimulus train during successful entrainment ( $E$ ) of the breathing cycle. Note that as stimulus (and entrainment) frequency increased, the phase angle of the stimulus train decreased. In conscious animals, the stimulus occurred at a significantly earlier phase in the respiratory cycle than in anesthetized rats. $\left({ }^{*} p<0.05\right)$.

In quiet conscious rats $(N=6)$, the breathing frequency (excluding sniffing and other behavioral artifacts) varied from $73 \pm$ 5 breaths $\cdot \min ^{-1}$ under hyperoxia to $151 \pm 8$ breaths $\cdot \min ^{-1}$ during exposure to $8 \% \mathrm{CO}_{2}$ (balance oxygen), corresponding to a range of $78 \pm 6$ breaths $\cdot \min ^{-1}$. Breathing frequencies as low as 25 breaths $\cdot \min ^{-1}$ were observed following light-induced hyperventilation, but such low frequencies were never observed under any other conditions. During periods of quiet breathing in these animals, RTN burst stimulation was able to entrain $\mathrm{fR}$ over a range of $22 \pm 5$ breaths/min, equivalent to $28 \pm 6 \%$ of the total operating range. In the case shown in Figure $2 B$, fR was entrained between 75 and 110 breaths $\cdot \min ^{-1}$ over more than 15 min despite brief behavioral disturbances. During periods of entrain- ment, a biphasic expiratory flow developed in all animals (Fig. 2B2-B4).

A scatter plot of the relationship between $\mathrm{fR}$ and stimulus frequency during respiratory pacing is shown in Figure $2 C$, corresponding to the cases shown in Figure 2, $A$ and $B$. At each set stimulus frequency, the instantaneous fR of 15-20 breaths were plotted (in $\mathrm{Hz}$ ). $\mathrm{fR}$ was reliably entrained at frequencies slightly greater than the resting frequency and tended to become more variable in relation to the stimulus frequency with increasing frequencies of entrainment. At the limit of the entrainable range $(>1 \mathrm{~Hz}$ in anesthetized rats and $>2 \mathrm{~Hz}$ in conscious rats), entrainment failed and breathing became arrhythmic (Fig. 2A3). 
A

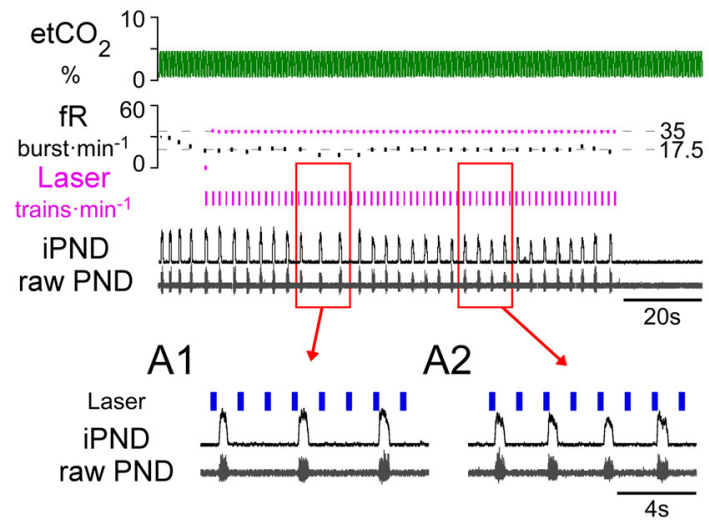

B

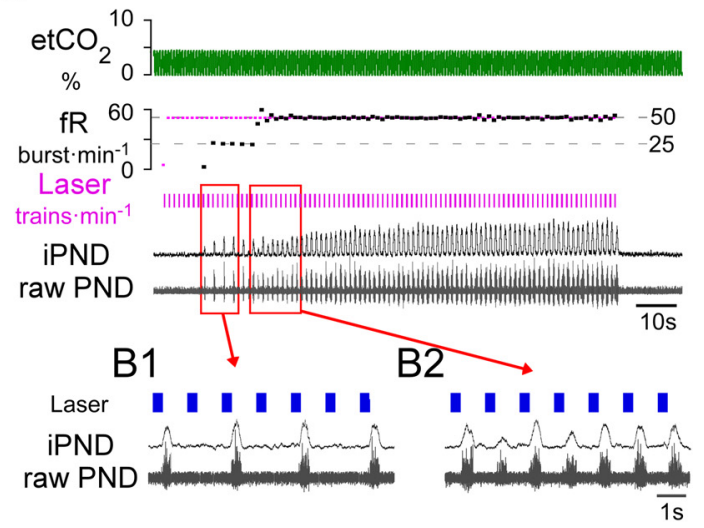

Figure 3. RTN photostimulation produces quantal breathing in urethane-anesthetized hypocapnic rats. $A$, When ventilation was adjusted to maintained et $\mathrm{C}_{2}$ close to the apneic threshold trains of laser light $\left(7 \times 5 \mathrm{~ms}\right.$ pulses at $20 \mathrm{~Hz}$; train frequency, $\left.35 \mathrm{~min}^{-1}\right)$ entrained PND in a 3:1 (A1), or 2:1 ratio (A2). $\boldsymbol{B}$, Quantal entrainment in a different anesthetized rat with et $\mathrm{C}_{2}$ maintained below apneic threshold. Laser trains $\left(7 \times 5 \mathrm{~ms}\right.$ pulse at $20 \mathrm{~Hz}$; train frequency, $\left.50 \mathrm{~min}^{-1}\right)$ delivered during phrenic apnea first produced quantal entrainment in a $2: 1$ ratio $(\boldsymbol{B} 1)$, eventually achieving 1:1 entrainment (B2).

Figure $2 D$ shows the grouped average frequency of a 15-20 breath segment during periods of entrainment under normocapnic conditions for all animals. The number of animals entrained at a given frequency is given by the numbers adjacent to each point. Entrainment in anesthetized and conscious rats was consistently observed at frequencies above resting fR. In anesthetized rats that responded particularly strongly to a 20 $\mathrm{Hz}$ stimulus (Fig. $1 \mathrm{~A}$ ), intermittent stimulation could entrain PND burst frequency over the entire operating range $(N=2$, Fig. $2 A, C$ ). Entrainment at average resting frequency was possible even in animals that showed relatively weak effects during continuous high-frequency stimulation. Entrainment was not possible at frequencies greater than the intrinsic maximum $\mathrm{fR}$ in anesthetized rats (i.e., $61 \pm 3$ bursts $\cdot \min ^{-1}$ ); stimulus frequencies equal to or above the maximum $\mathrm{fR}$ observed at high levels of $\mathrm{CO}_{2}$ produced arrhythmic PND bursting (Fig. 2A3). Entrainment also failed if the stimulus frequency was set below the resting $\mathrm{fR}$, but entrainment was possible at frequencies below the resting $\mathrm{fR}$ during periods of apnea (etCO $\mathrm{C}_{2}<4 \%$ ) in anesthetized rats (Fig. 3 ). In rats that were hyperventilated to maintain etCO ${ }_{2}$ slightly below the apneic threshold, RTN photostimulation produced periods of quantal entrainment in 6/7 animals (2:1 or 3:1 ratio, Fig. 3 ). Either high levels of et $\mathrm{CO}_{2}$ and/or high basal respiratory drive prevented entrainment in anesthetized animals.

In conscious rats the upper limit for entrainment was significantly lower than the maximum breathing rate ( $99 \pm 7$ vs $151 \pm$ 10 breaths $\left.\cdot \min ^{-1}, p<0.01\right)$, but in two cases entrainment was held at $>120$ breaths $\cdot \min ^{-1}$ in normocapnic conditions, and in one case we entrained $\mathrm{fR}$ at frequencies between 160 and 180 breaths $\cdot \min ^{-1}$ when inspired $\mathrm{CO}_{2}$ was elevated to $8 \%$.

Interestingly, during entrainment the photostimulus train always settled during mid- to late-expiration in both anesthetized and conscious rats (Fig. $2 E$ ). The train settled earlier during expiration as the entrainment frequency increased $(p<0.0001)$. Finally, the phase delay between the onset of the stimulus and inspiration was longer in conscious animals than in urethaneanesthetized animals $\left(229 \pm 7\right.$ vs $\left.244 \pm 11^{\circ}, p=0.0014\right)$, reflecting differences in neuronal excitability or some reorganization of the breathing network under anesthesia.
Prolonged entrainment of breathing at higher than baseline frequency causes compensatory decreases in tidal volume in conscious rats

Figure 4 shows the effect of prolonged respiratory pacing on tidal volume $\left(\mathrm{V}_{\mathrm{T}}\right)$ and total ventilation (MV) in a representative conscious rat. Light-driven hyperventilation $(3 \times 5 \mathrm{~ms}$ pulses at 20 $\mathrm{Hz}$; train frequency, 87 or $95 \mathrm{~min}^{-1}$ ) caused a progressive reduction in $\mathrm{V}_{\mathrm{T}}$ following the initial increase caused by the stimulus. This gradual reduction of $\mathrm{V}_{\mathrm{T}}$ caused $\mathrm{MV}$ to decline substantially, although without ever reaching the prestimulation level (Fig. $4 A, B)$. On average, when $\mathrm{fR}$ was entrained at $18 \pm 2$ breaths above baseline, there was a significant reduction in $\mathrm{V}_{\mathrm{T}}$ (from $2.2 \pm 0.3$ to $1.8 \pm 0.3 \mathrm{ml} ; p<0.01$ ) and MV (from $207 \pm 25$ to $\left.169 \pm 19 \mathrm{ml} \cdot \min ^{-1} ; p<0.05\right)$ relative to the early period of the entrainment (Fig. 4D). In these cases, $196 \pm 31$ s (range, 120-320 s) of entrainment was sufficient for $\mathrm{V}_{\mathrm{T}}$ to return to prestimulus values $(1.8 \pm 0.2$ vs $1.8 \pm 0.3 \mathrm{ml})$. MV was still elevated relative to baseline at the end of the entrainment period ( $169 \pm 19$ vs $139 \pm$ $\left.16 \mathrm{ml} \cdot \min ^{-1} ; p<0.05\right)$ due to the elevated breathing frequency caused by the stimulus $(p<0.01)$. At the end of the stimulus, $\mathrm{fR}$ and $\mathrm{V}_{\mathrm{T}}$ fell abruptly below resting values (Fig. $4 \mathrm{C}$ ). These aftereffects were most likely caused by hypocapnia given that MV was still significantly above resting values at the end of the stimulus.

\section{Active expiration is elicited during respiratory pacing in conscious normocapnic rats}

Under resting conditions, expiratory flow consists of a sharp initial peak (early-expiration) followed by a slow relaxation until the next inspiration (Fig. 5A). During pacing induced by RTN photostimulation at moderately elevated frequencies above resting values, expiratory flow became distinctly biphasic, the late component reflecting an observable contraction of abdominal and other thoracic expiratory muscles during late-expiration. As the frequency of entrainment was increased, the separation between early and late-expiratory flows became increasingly blurred and the two peaks fused into one (Fig. $5 A ; 95 \mathrm{~min}^{-1}$ ). Similarly, during hypercapnia ( $8 \% \mathrm{CO}_{2}$ added to oxygen) or continuous $20-23$ $\mathrm{Hz}$ photostimulation of the Phox $2 \mathrm{~b}$ neurons, there was no distinguishable separation between the early and late components of expiration (compare $20 \mathrm{~Hz}$ and $8 \% \mathrm{CO}_{2}$ in Fig. 5A). The transformation from a biphasic to a monophasic expiratory pattern 
A
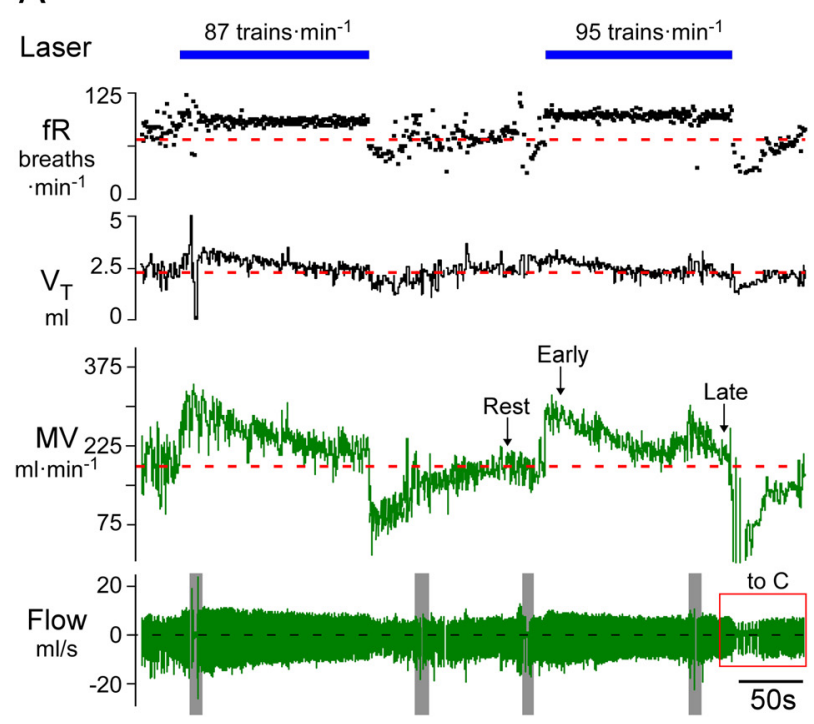

B

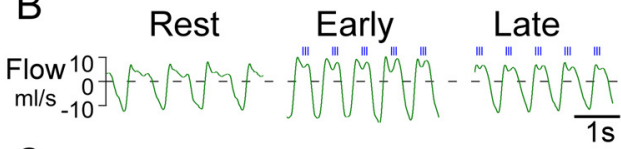

C

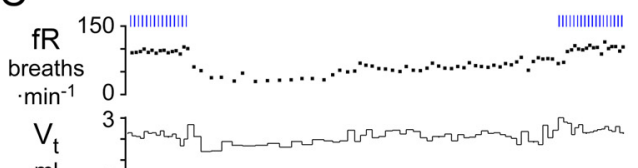

$\mathrm{ml} \quad 0^{3}$

Flow $\left.\begin{array}{r}10 \\ 0\end{array}\right]$ innhth

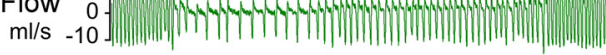

$\mathrm{D}$

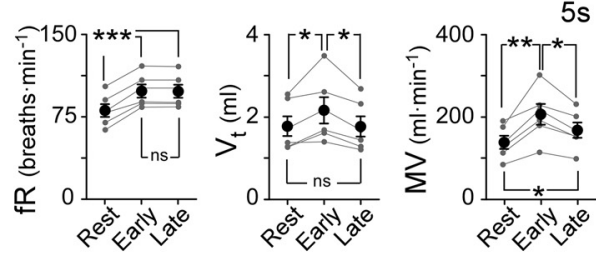

Figure 4. Respiratory pacing above resting frequency causes compensatory decreases in tidal volume in conscious rats. $A$, Conscious quiet rat breathing pure oxygen. Prolonged respiratory pacing $\left(3 \times 5 \mathrm{~ms}\right.$ pulses at $20 \mathrm{~Hz}$; train frequency, 87 and 95 trains $\left.\cdot \mathrm{min}^{-1}\right)$ caused a gradual reduction of the tidal volume, $V_{\mathrm{T}}$, to the resting (prestimulation) level, and a parallel decline in total ventilation, MV. After the end of the stimulus, tidal volume and frequency were transiently reduced, reflecting the hypocapnia caused by an incomplete return of MV to prestimulation levels. Gray bars in flow trace indicate behavioral disturbances. $\boldsymbol{B}$, Expanded traces from $\boldsymbol{A}$ showing the compensatory changes in $\mathrm{V}_{T}$ during long periods of entrainment $\left(147 \mathrm{~s}\right.$ at 95 trains $\cdot$ min $\left.{ }^{-1}\right)$. During the early part of the stimulation (middle, "Early"), $V_{T}$ was increased compared to the resting state (left). Two and a half minutes later (right, "Late"), $V_{T}$ had decreased to prestimulation levels. $C$, Expanded trace from $\boldsymbol{A}$ (red box) illustrating the after stimulus hypoventilation that followed $147 \mathrm{~s}$ of pacing at the rate of 95 trains $\min ^{-1}$. fR fell to 28 breaths $\cdot \min ^{-1}$ before returning to resting levels. $D$, Group data $(N=6)$ summarizing frequency of respiration (fR), tidal volume $\left(\mathrm{V}_{\mathrm{T}}\right)$ and total ventilation $(\mathrm{MV})$ at rest vs during the early and late part of the entrainment period $\left({ }^{* * *} p<0.001,{ }^{* *} p<0.01,{ }^{*} p<\right.$ 0.05; one-way ANOVA).

during continuous stimulation occurred during the first three

respiratory cycles (Fig. 5B), which suggests that active expiration is present during continuous activation of the RTN. To analyze this phenomenon, we divided the expiratory phase into two segments of equal duration (early-expiration and lateexpiration) and measured the relative volume of air expelled during late-expiration (late:early-expiratory ratio; Fig. 5C). During RTN photostimulated pacing, this ratio was much larger than at rest $(p<0.01$; Fig. $5 D)$. The increase in late-expiratory flow was also accompanied by an increase in tidal volume from $1.8 \pm 0.3$ to $2.2 \pm 0.3 \mathrm{ml}(p<0.01)$. At high $\mathrm{CO}_{2}$ $(8 \%)$ and during continuous high frequency photostimulation $(23 \mathrm{~Hz}, 30 \mathrm{~s})$, the late:early-expiratory ratio returned to values similar to those of resting conditions, in part reflecting an increase in peak flow during early-expiration $(20.2 \pm 4.1$ $\mathrm{ml} / \mathrm{s}$ during $23 \mathrm{~Hz} ; 23.0 \pm 4.1 \mathrm{ml} / \mathrm{s}$ during high $\mathrm{CO}_{2}$ ) compared to quiet conditions $(7.0 \pm 0.7 \mathrm{ml} / \mathrm{s} ; p<0.01)$ or photostimulated pacing at frequencies moderately above the resting rate $(7.7 \pm 1.2 \mathrm{ml} / \mathrm{s}$ for all entrainment frequencies; $p<0.01$ ) (Fig. 5C). These observations indicate that active expiration can be produced by activating the Phox $2 \mathrm{~b}$ neurons in conscious rats and does not require hypercapnia. However, active expiration can be observed by plethysmography only when the
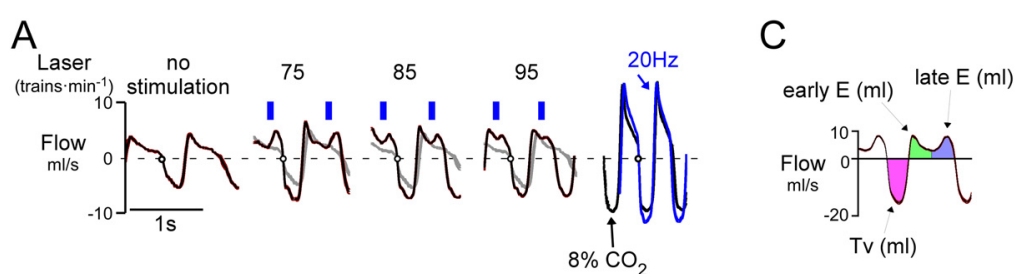

B
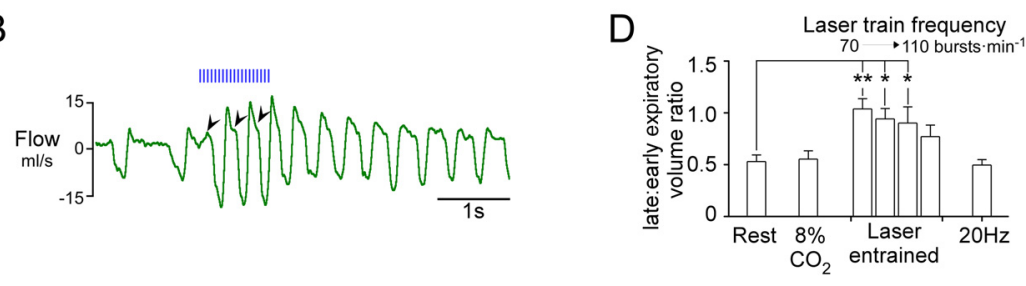

Figure 5. RTN photostimulation increases late-expiratory flow in conscious rats. A, Trajectory of respiratory flow (waveform average, $10-15$ breaths) at rest and during respiratory pacing caused by burst photostimulation of RTN at various frequencies (no stimulation, 75, 85 , and $95 \mathrm{~min}^{-1}$ ). Traces at extreme right depict flow trajectory during continuous $20 \mathrm{~Hz}$ stimulus (blue trace) or during hypercapnia $\left(8 \% \mathrm{CO}_{2}\right.$; black trace) in the same rat. At rest (left excerpt) during quiet breathing $\left(72\right.$ breaths $\cdot \min ^{-1}$ ) expiratory flow was monotonically decremental, reflecting the passive recoil of the lungs and no active expiration. When breathing was entrained at 75,85 , and 95 breaths $\cdot \min ^{-1}$ respectively (middle), a large positive peak in flow preceded inspiration. This peak was most prominent at lower frequencies of entrainment and tended to fuse with the early-expiratory peak at higher frequencies. During high-frequency continuous stimulation $(20 \mathrm{~Hz}$; right, blue) under hypercapnic conditions ( $8 \%$ inspired; right, black), the expiratory flow became monophasic, although active expiration must have been present. $\boldsymbol{B}$, Original record showing the effect of a $1 \mathrm{~s}$ stimulus train ( $20 \mathrm{~Hz}, 3 \mathrm{~ms}$ pulse). This example illustrates the progressive change in the pattern of expiratory flow from a biphasic to a monophasic pattern during continuous high-frequency photostimulation. $C$, Tidal volume, early and late-expiratory volumes are color coded; early and late volumes were defined by dividing expiration $(\mathrm{E})$ in two periods of equal duration. $\boldsymbol{D}$, Group data $(N=6)$. During stimulation-induced respiratory pacing, the ratio between late-expiratory and early-expiratory volume was significantly increased relative to resting breathing $\left({ }^{* *} p<0.01,{ }^{*} p<0.05\right.$ relative to rest; one-way ANOVA). The imbalance between late-expiratory and early-expiratory flow was most prominent at the lowest entrainment frequencies. When respiratory frequency was elevated by hypercapnia or continuous RTN stimulation at $20 \mathrm{~Hz}$, the ratio between late-expiratory and early-expiratory volume was no different from that observed at rest. 

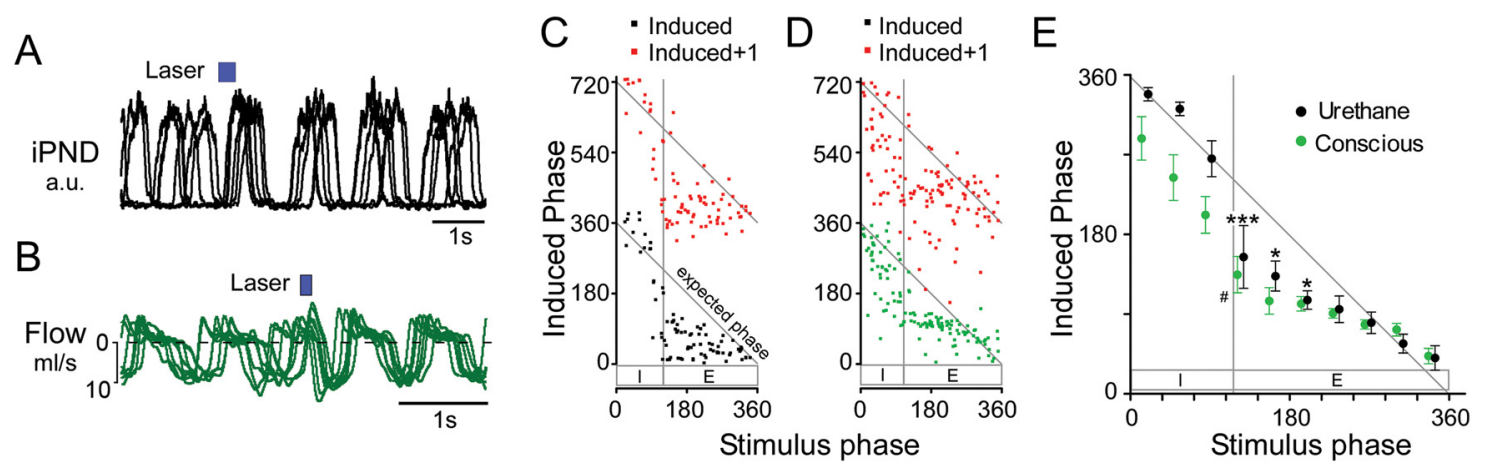

Figure 6. Low-frequency train stimulation resets the respiratory cycle. $A$, Overlaid recordings of integrated PND from one anesthetized rat. The waveforms are taken from periods during low-frequency $(<0.2 \mathrm{~Hz}$ ) train stimulation and are aligned to the onset of the stimulus (six traces in total). When the stimulus was delivered during expiration (in all cases), an advanced inspiratory burst occurred and the respiratory rhythm was reset as shown by the synchronization of PND activity following stimulation. $\boldsymbol{B}, 0$ verlaid flow traces from one conscious rat with description as per $\boldsymbol{A}$. $\boldsymbol{C}, \boldsymbol{D}$, Phase resetting curves from a single anesthetized $(\boldsymbol{C})$ and conscious rat $(\boldsymbol{D})$. In both cases, a stimulus delivered during expiration dramatically reduced the induced phase (stimulus onset to following inspiration) and reset the respiratory rhythm (i.e., $N+1$ induced phase $\left.\approx 360^{\circ}\right)$. $\boldsymbol{E}$, Grouped data from anesthetized $(N=4)$ and conscious experiments $(N=6)$ showing that stimulus-induced phase resetting occurs preferentially during early-expiration in anesthetized rats and conscious rats. Stimulation during late-inspiration did not significantly reset the respiratory rhythm in anesthetized animals or conscious rats ( ${ }^{* * *} p<0.001,{ }^{*} p<0.05$ vs $\mathrm{N}+1$ cycle in anesthetized rats; ${ }^{*} p<0.05$ vs $\mathrm{N}+1$ cycle in conscious rats two-way ANOVA).

breathing rate is modestly increased from rest. Active expiration is by definition present when breathing is dramatically increased by hypercapnia, but the compression of the expiratory phases combined with the large increase in early-expiratory flow rate produces a monophasic expiratory flow. Presumably for the same reasons, expiratory flow loses its biphasic characteristic during continuous stimulation of the Phox $2 \mathrm{~b}$ neurons. The important result is that continuous as opposed to intermittent stimulation of the Phox $2 \mathrm{~b}$ neurons stimulates breathing in a manner that is equally coordinated and at least as powerful as hypercapnia (Figs. $1 B, 5 A$ ), suggesting that intermittent activation of RTN neurons is not required for these neurons to produce an optimal breathing stimulation.

\section{Low-frequency train stimulation produces phase resetting of the respiratory cycle}

Electrical stimulation of a region coextensive with the RTN Phox $2 \mathrm{~b}$ neurons resets the respiratory rhythm in an in vitro brainstem-spinal cord preparation of neonatal rats (Onimaru and Homma, 2003). We examined whether selective photostimulation of the RTN Phox $2 \mathrm{~b}$ neurons can reset the breathing rate in anesthetized and conscious adult rats by applying single trains at very low rates $(0.05-0.1 \mathrm{~Hz})$ relative to the resting breathing frequency, such that each stimulus effectively occurred randomly during the breathing cycle, every 5-7 breathing cycles.

These stimuli invariably reset the respiratory rhythm in both anesthetized and conscious rats (Fig. 6A,B). The largest phase advances were observed when the onset of the stimulus coincided with early-expiration in both anesthetized and conscious rats with a modest or no phase advance when the stimulation occurred during early-inspiration. Importantly, the stimulus did not perturb the next respiratory cycle $(\mathrm{N}+1)$ (Fig. 6C,D). A modest lengthening of the respiratory cycle was observed in the anesthetized rats when the stimulus was delivered during inspiration, but this effect was solely due to a prolongation of the phrenic nerve discharge (i.e., $\mathrm{T}_{\mathrm{I}}$ ). In contrast, inspiratoryphase stimulation decreased total respiratory time in conscious rats (Fig. 6D,E).

We pursued this analysis with a more detailed examination of the phase-specific perturbations in inspiratory and expiratory time, tidal volume (conscious rats), or PND amplitude (anesthesia) (Fig. $7 A-D)$. Consistent with our phase-resetting analysis, brief trains of stimuli invariably produced a phase advance (i.e., $\mathrm{T}_{\text {Tot }}$ reduction) relative to the natural cycle (type 1 phase response; Fig. $7 A, B, E)$. In conscious $(N=5)$ and anesthetized rats $(N=4)$, phase advances were entirely due to a reduction in $\mathrm{T}_{\mathrm{E}}$ (Fig. $7 F$ ). Significant increases in $\mathrm{T}_{\mathrm{E}}$ were never observed. In conscious rats, stimuli applied between mid-inspiration and mid-expiration produced the largest reductions in both $\mathrm{T}_{\mathrm{Tot}}$ (31 $\pm 7 \%$ during late-inspiration, $p<0.0001$; Fig. $7 E$ ) and $\mathrm{T}_{\mathrm{E}}$ (41 $\pm 11 \%$ during late-inspiration, $p<0.001$; Fig. $7 F$ ) whereas, stimuli applied in mid-expiration caused the greatest reductions in $\mathrm{T}_{\mathrm{Tot}}$ in anesthetized rats $(16 \pm 4 \%, p<0.001$; Fig. $7 E)$ and $\mathrm{T}_{\mathrm{E}}$ $(25 \pm 5 \%, p<0.001$; Fig. $7 F)$. In anesthetized rats, $\mathrm{T}_{\text {Tot }}$ was unchanged when stimuli occurred during inspiration, despite modest shortening of $\mathrm{T}_{\mathrm{E}}$ (Fig. $7 E, F$ ), because $\mathrm{T}_{\mathrm{I}}$ was significantly increased ( $12.3 \pm 0.4 \%$ during late-inspiration, $p<0.001$; Fig. $7 G)$. In contrast, stimuli delivered during inspiration produced no significant perturbation of $\mathrm{T}_{\mathrm{I}}$ in conscious rats, and we never observed a significant shortening of $T_{I}$ in either the anesthetized or conscious conditions regardless of the stimulus phase (Fig. 7G,I)

Stimuli delivered during inspiration did not produce a statistically significant change in $\mathrm{V}_{\mathrm{T}}$ or PND amplitude of the targeted respiratory phase ( $N$ th cycle; Fig. $7 H$ ), but a clear increase was produced in two anesthetized and two conscious rats, which had the most robust activation of breathing during high-frequency photostimulation (for example, Fig. $7 A, B$ ). In contrast, when the stimulus occurred during late-expiration in conscious rats, $\mathrm{V}_{\mathrm{T}}$ of the following inspiration was consistently increased $(26 \pm 3 \%$, $p<0.05$; Fig. $7 J)$. This augmented inspiratory effort was preceded by a marked increase in late-expiratory flow similar in every detail to that observed during respiratory pacing (Fig. 7B3B4). In anesthetized rats, there was also a tendency for PND amplitude to be greater if the stimulus fell during late-expiration (Fig. $7 J$ ) but the change was not statistically significant in four rats. These results suggest that $V_{T}$ is most efficiently increased by intermittent stimulation of the Phox $2 \mathrm{~b}$ neurons when the stimuli occur during mid- to late-expiration and trigger a prior forced expiration. 
A

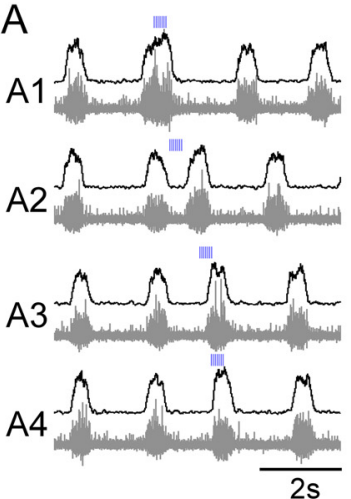

B

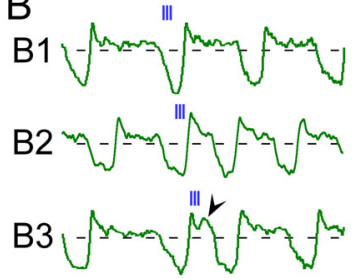

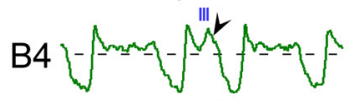

B5 7 frong

$\frac{10 \mathrm{ml} / \mathrm{s}}{1 \mathrm{~s}}$

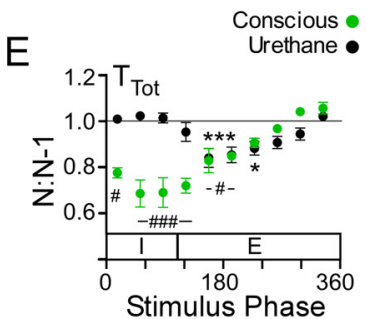

G

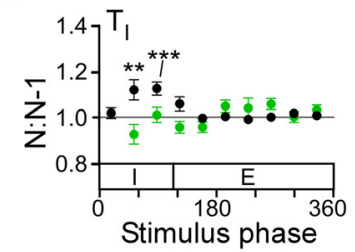

I

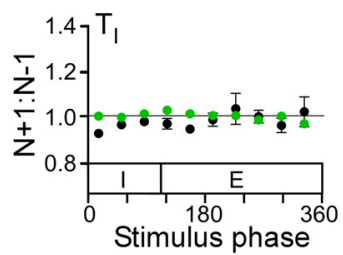

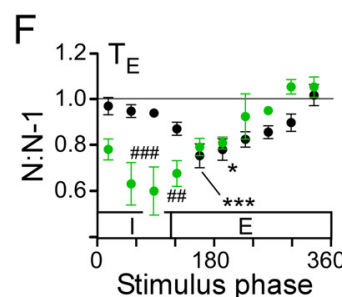

$\mathrm{H}$

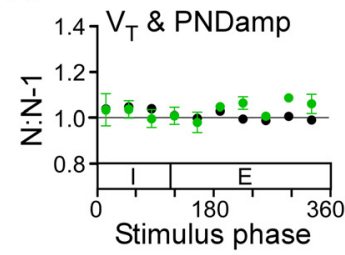

$\mathrm{J}$

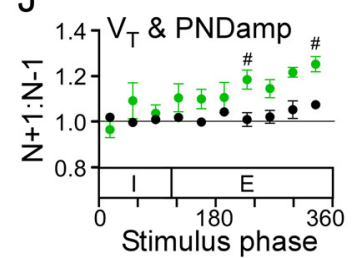

Figure 7. Phase-dependent perturbations of respiratory frequency and amplitude in anesthetized and conscious rats. RTN photostimulation was applied at frequencies less than the resting breathing frequency $(<0.2 \mathrm{~Hz}$ ) so that the stimulus occurred at random within the breathing cycle. $\boldsymbol{A}$, Original recordings of integrated (top traces) and raw PND (bottom traces) in an anesthetized rat. Train stimulation produced phase-specific perturbations in total respiratory cycle $\left(T_{T o t}\right)$, inspiratory time $\left(T_{1}\right)$, expiratory time $\left(T_{E}\right)$, and peak PND amplitude $(P N D a m p)$. In this example, stimulation during late-inspiration (A1) increased PNDamp and $\mathrm{T}_{\mathrm{I}}$. When trains occurred during expiration $(\boldsymbol{A 2}, \boldsymbol{A 3})$ there was a decrease in $\mathrm{T}_{\mathrm{E}}$ with a modest increase in PNDamp, but no change in $\mathrm{T}_{1}$. When the stimulus coincided with the spontaneous phrenic burst, PNDamp was modestly increased and there was no change in respiratory timing $(\boldsymbol{A} 4)$. $\boldsymbol{B}$, Respiratory flow in a conscious rat. As in $\boldsymbol{A}$, brief trains ( 3 pulses at $20 \mathrm{~Hz}$ ) produced phase-specific perturbations in $\mathrm{T}_{\mathrm{Tot}}, \mathrm{T}_{\mathrm{l}}, \mathrm{T}_{\mathrm{E}}$, and tidal volume, $\mathrm{V}_{\mathrm{T}}$. Stimulation during early-inspiration increased $\mathrm{V}_{\mathrm{T}}(\boldsymbol{B} 1$ ). Stimulation during lateinspiration reduced the expiratory phase $(\boldsymbol{B} 2)$. Stimulation during early- to mid-expiration decreased $\mathrm{T}_{\mathrm{E}}$ and produced active expiration $(\boldsymbol{B} \mathbf{3}, \boldsymbol{B} 4)$. Stimulation during late-expiration $(\boldsymbol{B} 5)$ shortened $\mathrm{T}_{\mathrm{E}}$ modestly and increased $\mathrm{V}_{\mathrm{T}}$ of the next inspiration. $\boldsymbol{C}, \boldsymbol{D}$, Data analysis from experiments in anesthetized ( $\boldsymbol{C}$ ) and conscious (D) rats. See Materials and Methods for more details. $\boldsymbol{E}-\boldsymbol{J}$, Group data from anesthetized $(N=4)$ and conscious experiments $(N=5)$. $\boldsymbol{E}$, Effect of stimulus on respiratory period $\left(\mathrm{T}_{\mathrm{Tot}}\right) . \boldsymbol{F}$, Effect of stimulus on expiratory duration ( $\left.\mathrm{T}_{\mathrm{E}}\right)$. $\boldsymbol{G}$, Effect of stimulus on inspiratory duration $\left(\mathrm{T}_{1}\right)$. $\boldsymbol{H}$, Effect of stimulus on tidal volume or PNDamp. In $\boldsymbol{E}-\boldsymbol{H}$, values are expressed as ratio between respiratory cycles $\mathrm{N}$ and $\mathrm{N}-1$ (see definitions in $\boldsymbol{C}$ and $\boldsymbol{D}$ ). A value of 1 indicates no change from the $\mathrm{N}-1$ (control) cycle. Note that in both conditions we did not observe significant increases in $\mathrm{T}_{\mathrm{E}}$. $I$, J, Group data from anesthetized $(N=4)$ and conscious experiments $(N=5)$ showing the changes in PNDamp or $\mathrm{V}_{\mathrm{T}}$ in the $\mathrm{N}+1$ cycle relative to the control cycle $\mathrm{N}-1$. Brief stimulation during late-expiration significantly increased $\mathrm{V}_{\mathrm{T}}$ but not $\mathrm{PNDamp}$ of the following inspiratory effort (N +1 cycle) $\left({ }^{* * *} p<0.001,{ }^{*} p<0.05\right.$ vs N +1 for $\boldsymbol{E}-\boldsymbol{H}$ and N +2 for $\boldsymbol{I}, \boldsymbol{J}$ in anesthetized rats; ${ }^{\# \#} p<0.001,{ }^{\# \#} p<0.01,{ }^{\#} p<0.05$ vs N +1 for $\boldsymbol{E}-\boldsymbol{H}$ and $\mathrm{N}+2$ for $\boldsymbol{I}, \boldsymbol{J}$ in conscious rats).

\section{Histology}

Phox 2 b immunoreactivity was observed in $98.3 \%$ of the mCherry fluorescent neurons (four transverse sections counted from each of three rats), demonstrating that the virus transfected Phox $2 \mathrm{~b}$ neurons with a high degree of selectivity (Fig. $8 B$ ). ChR2mCherry was detectable only in neurons, and the cell distribution was confined to the region of the rostral ventrolateral medulla that harbors Phox2b-expressing neurons (Stornetta et al., 2006; Kang et al., 2007; Abbott et al., 2009a). The transfected population consisted of a mixture of catecholaminergic neurons, noncatecholaminergic neurons, and a very small number of cholinergic neurons (Figure $8 A$ ).

On average (seven anesthetized and five conscious rats), ChR2-mCherry was detected in $383 \pm 50$ catecholaminergic neurons, $13.2 \pm 3.5$ cholinergic neurons, and $255.9 \pm 35.5$ noncatecholaminergic, noncholinergic (presumptive RTN-Phox2b) neurons per rat. These figures represent the number of neurons counted in a one-in-six series of sections multiplied by six. Given our prior estimate of about 2000 RTN-Phox $2 \mathrm{~b}$ neurons per rat, between 5.4 and $26.7 \%$ (median $13.8 \%$ ) of the total population of RTN-Phox2b neurons could have been directly photoactivated.

The two rats that had received spinal injections of antidopamine $\beta$-hydroxylase-conjugated saporin toxin had many fewer catecholaminergic ChR2-labeled neurons as expected (Abbott et al., 2009a) (168 and 162, respectively, per rat), but they had an average number of RTN-Phox2b neurons (234 and 474 , respectively). The respiratory responses elicited by photostimulation in these animals were not different from the response in animals with an intact $\mathrm{C} 1$ population, confirming prior observations that, under our experimental conditions, these responses likely result from activation of the RTNPhox $2 b$ neurons rather than the catecholaminergic neurons (Abbott et al., 2009a).

The brain distribution of the transfected neurons is represented in Figure $8 D$. This panel depicts the superimposed locations of all the transfected neurons identified in 12 cases (five conscious and seven anesthetized). The catecholaminergic neurons are all, by definition, Phox $2 \mathrm{a}$ or b containing. At the rostrolateral edge of the facial motor nucleus, these neurons are predominantly of the A5 variety (Byrum and Guyenet, 1987). Medially, they belong to the $\mathrm{C} 1$ adrenergic cell group. Of note, the lateral region situated between the spinal trigeminal tract and nucleus ambiguus, which may contain an expiratory oscillator (Pagliardini et al., 2011), had very few ChR2-expressing neurons and, as indicated above, the overwhelming majority of these cells in our cases were Phox $2 \mathrm{~b}$ positive. 

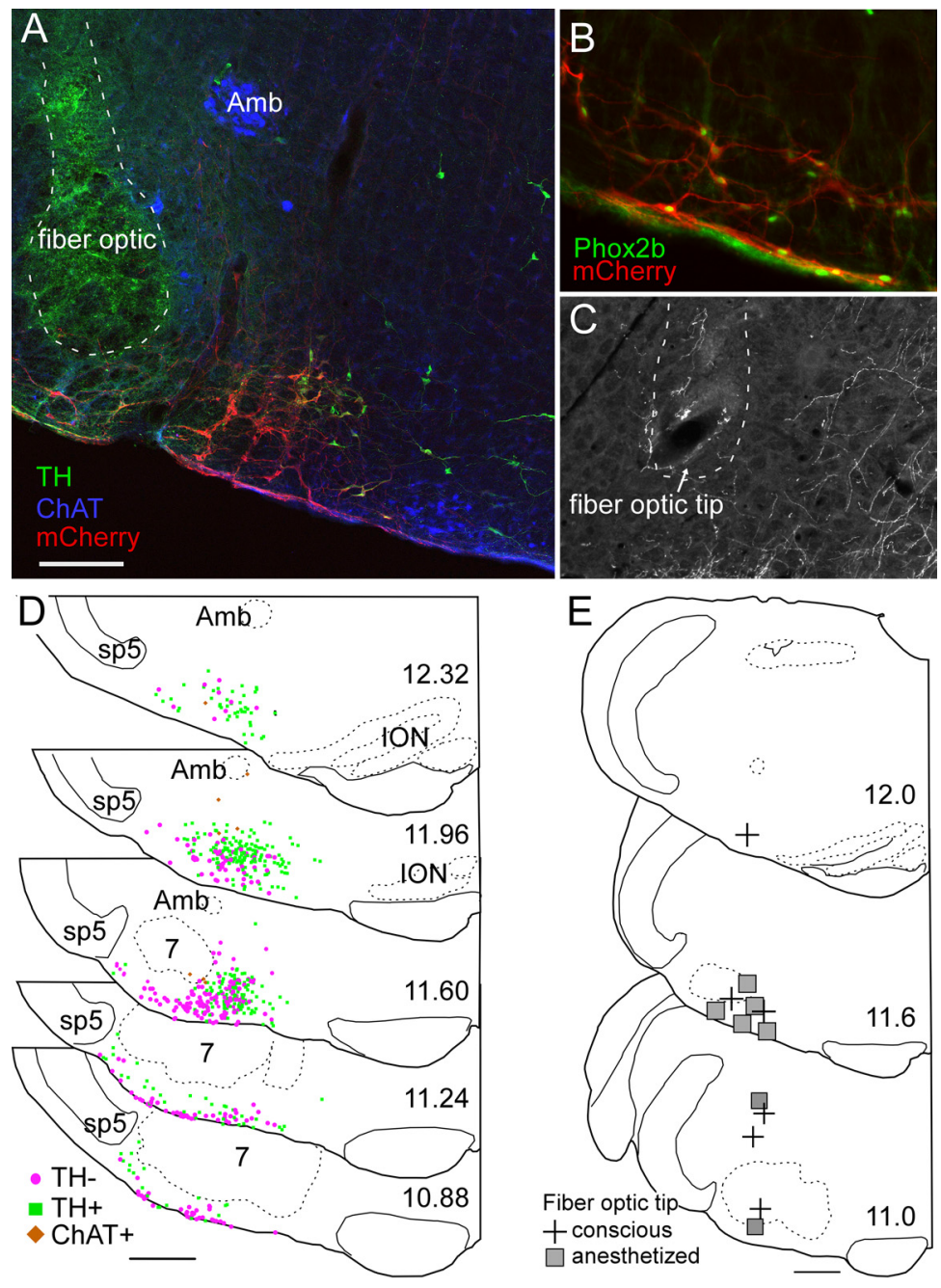

Figure 8. Anatomical distribution of ChR2-transfected cells and fiber optic locations. A, Example of fiber optic location (dotted line, the green color within the fiber optic tract is an auto fluorescence artifact) next to the ChR2-mCherry-transfected neurons (mCherry in red). Choline acetyl-transferase-immunoreactive neurons (represented in blue) located in the ambiguus nucleus $(\mathrm{Amb})$ and medial to the retrotrapezoid nucleus are not transfected. Several mCherry-positive neurons in this transverse section are catecholaminergic (tyrosine hydroxylase, $\mathrm{TH}$, in green). These neurons generally occupy a medial location relative to the noncatecholaminergic, noncholinergic neurons (the RTN-Phox2b neurons). $\boldsymbol{B}$, Native mCherry fluorescence (red) detected along with Phox2b-immunoreactivity (green nuclei). Note the presence of Phox2b in every transfected neuron, including within the marginal layer of the retrotrapezoid nucleus. $\boldsymbol{C}$, Example of a fiber optic tract (dotted line) found to end next to the labeled axons of ChR2-mCherry-transfected neurons. $\boldsymbol{D}$, Caudal-to-rostral series of transverse sections (bregma levels in millimeters as indicated) depicting the location of all the ChR2-mCherry-transfected neurons identified in 12 cases. Computer-generated drawings with each ChR2-mCherry-transfected neuron indicated by either a green square ( $\mathrm{TH}+, \mathrm{TH}$-immunoreactive), brown diamond (ChAT+, choline acetyl transferase-immunoreactive), or magenta circle (TH-, neither TH-immunoreactive nor ChAT-immunoreactive) from the respective sections indicated for each bregma level from 12 animals were optimally superimposed using landmarks such as the ventral medullary surface, the edge of the trigeminal tract, the compact portion of nucleus ambiguus, the inferior olive, and the facial motor nucleus. $\boldsymbol{E}$, Location of fiber optic tips plotted on three transverse sections at the indicated bregma level (in $\mathrm{mm}$ ). Scale bars: (in $\boldsymbol{A}) \boldsymbol{A}, \boldsymbol{C}, 200 \mu \mathrm{m}, \boldsymbol{B}, 100 \mu \mathrm{m}$; (in $\boldsymbol{D}, \boldsymbol{E}), 500 \mu \mathrm{m}$.

The location of the fiber optic tips (13 rats) is represented in Figure $8 E$. The fiber optic tips were generally very close to the RTN, but in several cases in which strong respiratory stimulation was elicited even with brief stimuli, the tip of the fiber optic was at a considerable distance $(>1 \mathrm{~mm})$ from the somata of the ChR2expressing neurons. In such cases, the fiber optic tip was invariably close $(<250 \mu \mathrm{m})$ to the main axons of these cells (Fig. $8 C$ ), which take an initial mediodorsal course through the intermediate reticular nucleus (IRt, after Paxinos and Watson, 1986; Abbott et al., 2009a). Therefore, we presume that neuronal activation must have often resulted from stimulating the axons of the ChR2expressing axons.

\section{Discussion}

Summarizing the new results, selective activation of RTN-Phox $2 \mathrm{~b}$ neurons increases the breathing rate, inspiratory amplitude, and active expiration; these effects are occluded by hypercapnia. Phasic activation of RTN-Phox $2 b$ neurons resets and entrains the breathing cycle and produces an expiration-inspiration sequence. When frequency is fixed, ventilatory homeostasis is partially restored via tidal volume reduction.

Although phasic activation of RTNPhox $2 \mathrm{~b}$ neurons recruits both inspiratory and expiratory mechanisms, continuous activation produces far more powerful and yet effectively coordinated breathing stimulation.

Histology and experimental limitations ChR2 was expressed by Phox2b-containing neurons consisting of catecholaminergic and noncatecholaminergic (RTN) neurons (Abbott et al., 2009a). Few ChR2-transfected neurons were within the lateral parafacial region where others located an expiratory oscillator (Pagliardini et al., 2011), except at the medullary surface under the facial motor nucleus, where almost every neuron is Phox2b-positive (Takakura et al., 2008).

Destruction of most of the spinally projecting catecholaminergic $\mathrm{C} 1 \mathrm{neu}-$ rons did not affect the breathing response to photostimulation of RTNPhox $2 \mathrm{~b}$ neurons in anesthetized rats, consistent with our previous observations (Abbott et al., 2009a). However, our results cannot exclude a contribution of other ChR2-expressing C1 neurons, perhaps via their projections to the RTN (Card et al., 2006; Rosin et al., 2006).

We postulate that the observed breathing stimulation resulted from direct activation of the brainstem respiratory network by RTN neurons, but we cannot exclude indirect effects on breathing mediated by changes in vigilance or cortical function (Boon et al., 2004; Buchanan and Richerson, 2010).

RTN-induced pacing and resetting of the breathing rate Electrical stimulation of the RTN entrains and resets the respiratory rhythm in neonates in vitro (Onimaru and Homma, 2003). As shown here, selective activation of the Phox $2 b$ neurons achieves the same result in conscious adult rats. This entrainment occurred over a limited range of breathing frequencies, possibly because only a small fraction $(\sim 14 \%)$ of the RTN neurons expressed ChR2. Phox $2 \mathrm{~b}$ neuron stimulation produced quantal breathing only in anesthetized rats maintained slightly below apneic threshold, consistent with the fact that this pattern is typically observed when the respiratory network is in a low excitability state (Mellen et al., 2003; Ballanyi et al., 2009). During 
low frequency stimulation, the greatest phase advance occurred when Phox $2 \mathrm{~b}$ neurons are stimulated during early- and midexpiration, probably because these neurons can most productively activate pre-I/I "rhythmogenic" and early-I inhibitory neurons during this time window, thereby initiating a premature inspiration (Rubin et al., 2009). Similarly, during pacing the respiratory rhythm reset and the stimulus settled during mid- to late-expiration. Respiratory entrainment by somatic afferent stimulation has the same characteristic, which has been explained by inhibition of late-expiratory neurons via the parabrachial nucleus (Potts et al., 2005). A similar mechanism could partly account for the present results, because RTN neurons densely innervate the dorsal pons (Abbott et al., 2009a; Bochorishvili et al., 2011).

Photostimulation of unidentified parafacial neurons produced respiratory rhythm resetting and terminated inspiration in anesthetized, vagotomized, spontaneously breathing rats, but such stimulation produced relatively small increases in inspiratory amplitude and frequency (Pagliardini et al., 2011). In our anesthetized, ventilated preparation, brief stimulation of Phox $2 b$ neurons during inspiration actually prolonged $\mathrm{T}_{\mathrm{I}}$, suggesting that RTN neurons can delay the I-E transition in the absence of lung stretch receptor feedback. More importantly, with or without anesthesia, continuous activation of the Phox $2 \mathrm{~b}$ cells increased tidal volume and markedly increased respiratory rate (see also Kanbar et al., 2010) as expected from a cell group that contributes to the effects of hypercapnia. These differences support the conclusion of Pagliardini et al. (2011) that the effects seen in their experiments were not primarily caused by stimulating the Phox2b neurons.

As observed previously, continuous $20 \mathrm{~Hz}$ activation of the RTN-Phox2b neurons under anesthesia produces a breathing activation that decays very slowly upon cessation of the stimulus (Abbott et al., 2009a; Fig. 2). Yet, we show that RTN-Phox2b neurons can also influence breathing on a breath-to-breath basis. RTN neurons may therefore use both fast ionotropic and slow metabotropic transmission. Indeed, the ultrastructure of RTNPhox $2 \mathrm{~b}$ synapses resembles classical non-NMDA glutamatergic synapses (Bochorishvili et al., 2011), but the long afterdischarge elicited by high-frequency photostimulation and the attenuation of the central chemoreflex by certain metabotropic glutamate receptor antagonists (Takakura et al., 2011) suggest additional metabotropic mechanisms. A long afterdischarge also occurs following tonic and phasic electrical activation of peripheral chemoreceptors and muscle afferents. This phenomenon is therefore not uniquely associated with RTN activation and may be a property of the rhythm and pattern generator (Eldridge et al., 1981).

\section{Chemoreflex regulation of breathing during RTN-induced pacing}

The respiratory network allows independent changes of frequency and amplitude under many conditions such as panting, sniffing, emotions, and vocalization/speech. As shown here, when breathing frequency is fixed above resting levels in conscious rats, tidal volume gradually decreases. In conscious man, tidal volume compensates for volitional breathing at elevated frequencies so that alveolar ventilation remains essentially constant (Haouzi and Bell, 2009). These data and ours indicate that the amplitude component of the central chemoreflex can be automatically adjusted somewhat independently of the breathing frequency to maintain $\mathrm{CO}_{2}$ homeostasis. RTN-Phox2b neurons innervate both the region that contains phrenic premotor neu- rons as well as the Pre-Bötzinger complex (Abbott et al., 2009a; Bochorishvili et al., 2011). These projections may underlie their ability to regulate tidal volume independently of respiratory frequency.

\section{RTN-Phox2b neurons trigger active expiration in conscious rats}

Active expiration is the recruitment of expiratory muscles for breathing which occurs predominantly during exercise, hypoxia, or hypercapnia (Iscoe, 1998). Janczewski and Feldman (2006) first showed that the RTN region contributes to active expiration, but opinions vary regarding the role of RTN-Phox $2 b$ neurons in this process (Marina et al., 2010; Pagliardini et al., 2011). Our results, obtained using conscious rats, support prior evidence that the Phox $2 b$ neurons drive active expiration (Marina et al., 2010). We add that an orderly expiration-inspiration sequence is triggered by a brief activation of these cells, even in normocapnia. The control of expiratory muscles by RTN-Phox $2 \mathrm{~b}$ neurons is consistent with their postulated function of increasing lung ventilation according to the intensity of the hypercapnic stimulus (Guyenet et al., 2010). Our experiments do not clarify whether expiration, breathing rate, and inspiration are regulated by the same Phox2b neurons (Abdala et al., 2009b; Marina et al., 2010). However, in conscious rats active expiration was produced by activating RTN-Phox $2 b$ neurons during mid-expiration rather than late-expiration, i.e., considerably earlier than when the neurons assumed to drive abdominal muscles are active (Abdala et al., 2009a; Pagliardini et al., 2011), suggesting that the latter neurons are downstream from the Phox $2 \mathrm{~b}$ cells. These neurons may reside in the lateral medullary region identified by Pagliardini et al. (2011).

Brief photostimulation of the Phox $2 \mathrm{~b}$ neurons never increased $\mathrm{T}_{\mathrm{E}}$ in conscious or anesthetized adult rats. Accordingly, the pacemaker handshake synchronization mechanism of mammalian respiratory rhythmogenesis (Wittmeier et al., 2008) does not account for the production of an expiration-inspiration sequence by mature Phox $2 \mathrm{~b}$ neurons.

\section{Phasic activation of Phox $2 \mathrm{~b}$ neurons is not necessary to drive breathing}

Under normocapnic conditions the discharge of mature RTNPhox $2 b$ neurons is tonic, but with hypercapnia their discharge becomes both more intense and increasingly synchronized with respiration (Guyenet et al., 2005; Moreira et al., 2007). If phasic stimulation of RTN-Phox $2 b$ neurons was required to produce strong and optimally coordinated contractions of inspiratory and expiratory muscles, then tonic stimulation should produce arrhythmic and inefficient breathing and phasic stimulation should produce the greatest increases in lung ventilation. We observed the exact opposite. Continuous high-frequency activation of RTN-Phox $2 \mathrm{~b}$ neurons produced the largest increases in ventilation, reflecting the effective coordination of both inspiratory and expiratory respiratory muscles in conscious animals, whereas phasic activation tended to cause a breakdown of the breathing rhythmicity when applied at high frequency. Therefore, as proposed previously (Guyenet et al., 2005), the effectiveness of the RTNPhox $2 b$ neurons is presumably encoded by their mean discharge rate rather than by their pattern, and their respiratory synchronization during hypercapnia may simply be the manifestation of an inhibitory feedback that emanates from phasically active respiratory neurons and protects the respiratory system from excessive stimulation. The patterning of the 
muscle contractions elicited by $\mathrm{CO}_{2}$ via the activation of the RTN-Phox $2 b$ neurons presumably relies on the downstream respiratory pattern generator, which likely includes the parafacial region (Pagliardini et al., 2011). Expected targets of the RTN-Phox $2 b$ neurons include early-I and preI-I neurons whose coordinated excitation is presumably required to increase the breathing rate (Rubin et al., 2009), post-I neurons (Marina et al., 2010) that restrain the duration of the phrenic nerve discharge, and premotor neurons whose activation may explain why the chemoreflex can still operate when breathing frequency is fixed ((Haouzi and Bell, 2009); present data).

\section{Conclusions}

Consistent with their anatomical projections, RTN-Phox2b neurons regulate lung ventilation by recruiting inspiratory and expiratory muscles and regulating the breathing rate. Intermittent activation of adult RTN-Phox $2 \mathrm{~b}$ neurons entrains the respiratory rhythm, but their tonic activation produces far greater breathing stimulation. While a rhythmogenic role in adulthood cannot be ruled out, the average discharge rate of these neurons seems to be the critical factor for their role in breathing regulation.

\section{References}

Abbott SB, Stornetta RL, Fortuna MG, Depuy SD, West GH, Harris TE, Guyenet PG (2009a) Photostimulation of retrotrapezoid nucleus Phox2b-expressing neurons in vivo produces long-lasting activation of breathing in rats. J Neurosci 29:5806-5819.

Abbott SB, Stornetta RL, Socolovsky CS, West GH, Guyenet PG (2009b) Photostimulation of channelrhodopsin-2 expressing ventrolateral medullary neurons increases sympathetic nerve activity and blood pressure in rats. J Physiol 587:5613-5631.

Abdala AP, Rybak IA, Smith JC, Paton JF (2009a) Abdominal expiratory activity in the rat brainstem-spinal cord in situ: patterns, origins, and implications for respiratory rhythm generation. J Physiol 587:3539-3559.

Abdala AP, Rybak IA, Smith JC, Zoccal DB, Machado BH, St-John WM, Paton JF (2009b) Multiple pontomedullary mechanisms of respiratory rhythmogenesis. Respir Physiol Neurobiol 168:19-25.

Ballanyi K, Ruangkittisakul A, Onimaru H (2009) Opioids prolong and anoxia shortens delay between onset of preinspiratory (pFRG) and inspiratory (preBotC) network bursting in newborn rat brainstems. Pflugers Arch 458:571-587.

Bochorishvili G, Coates MB, Stornetta RL, Guyenet PG (2011) PreBötzinger complex receives glutamatergic innervation from galaninergic and other retrotrapezoid nucleus neurons. J Comp Neurol. Advance online publication. Retrieved Sept 20, 2011. doi:10.1002/cne.22769.

Boon JA, Garnett NB, Bentley JM, Milsom WK (2004) Respiratory chemoreflexes and effects of cortical activation state in urethane anesthetized rats 8. Respir Physiol Neurobiol 140:243-256.

Buchanan GF, Richerson GB (2010) Central serotonin neurons are required for arousal to CO2. Proc Natl Acad Sci U S A 107:16354-16359.

Byrum CE, Guyenet PG (1987) Afferent and efferent connections of the A5 noradrenergic cell group in the rat. J Comp Neurol 261:529-542.

Card JP, Sved JC, Craig B, Raizada M, Vazquez J, Sved AF (2006) Efferent projections of rat rostroventrolateral medulla $\mathrm{C} 1$ catecholamine neurons: Implications for the central control of cardiovascular regulation. J Comp Neurol 499:840-859.

Drorbaugh JE, Fenn WO (1955) A barometric method for measuring ventilation in newborn infants 1 . Pediatrics 16:81-87.

Eldridge FL, Gill-Kumar P, Millhorn DE (1981) Input-output relationships of central neural circuits involved in respiration in cats. J Physiol 311:81-95.

Feldman JL (2011) Chapter 14-looking forward to breathing 4. Prog Brain Res 188:213-218.

Fortuna MG, Stornetta RL, West GH, Guyenet PG (2009) Activation of the retrotrapezoid nucleus by posterior hypothalamic stimulation. J Physiol 587:5121-5138

Goridis C, Brunet JF (2010) Central chemoreception: lessons from mouse and human genetics. Respir Physiol Neurobiol 173:312-321.

Gourine AV, Kasymov V, Marina N, Tang F, Figueiredo MF, Lane S, Tesche- macher AG, Spyer KM, Deisseroth K, Kasparov S (2010) Astrocytes Control Breathing Through pH-Dependent Release of ATP. Science 329:571-575.

Guyenet PG, Mulkey DK, Stornetta RL, Bayliss DA (2005) Regulation of ventral surface chemoreceptors by the central respiratory pattern generator. J Neurosci 25:8938-8947.

Guyenet PG, Stornetta RL, Bayliss DA (2010) Central respiratory chemoreception. J Comp Neurol 518:3883-3906.

Haouzi P, Bell HJ (2009) Control of breathing and volitional respiratory rhythm in humans. J Appl Physiol 106:904-910.

Hwang DY, Carlezon WA Jr, Isacson O, Kim KS (2001) A high-efficiency synthetic promoter that drives transgene expression selectively in noradrenergic neurons. Hum Gene Ther 12:1731-1740.

Iscoe S (1998) Control of abdominal muscles. Prog Neurobiol 56:433-506. Janczewski WA, Feldman JL (2006) Distinct rhythm generators for inspiration and expiration in the juvenile rat. J Physiol 570:407-420.

Kanbar R, Stornetta RL, Cash DR, Lewis SJ, Guyenet PG (2010) Photostimulation of Phox $2 \mathrm{~b}$ medullary neurons activates cardiorespiratory function in conscious rats. Am J Respir Crit Care Med 182:1184-1194.

Kang BJ, Chang DA, Mackay DD, West GH, Moreira TS, Takakura AC, Gwilt JM, Guyenet PG, Stornetta RL (2007) Central nervous system distribution of the transcription factor Phox $2 \mathrm{~b}$ in the adult rat. J Comp Neurol 503:627-641.

Lazarenko RM, Milner TA, Depuy SD, Stornetta RL, West GH, Kievits JA, Bayliss DA, Guyenet PG (2009) Acid sensitivity and ultrastructure of the retrotrapezoid nucleus in Phox2b-EGFP transgenic mice. J Comp Neurol 517:69-86.

Li A, Nattie E (2002) CO2 dialysis in one chemoreceptor site, the RTN: stimulus intensity and sensitivity in the awake rat. Respir Physiol Neurobiol 133:11-22.

Marina N, Abdala AP, Trapp S, Li A, Nattie EE, Hewinson J, Smith JC, Paton JF, Gourine AV (2010) Essential role of Phox2b-expressing ventrolateral brainstem neurons in the chemosensory control of inspiration and expiration. J Neurosci 30:12466-12473.

Mellen NM, Janczewski WA, Bocchiaro CM, Feldman JL (2003) Opioidinduced quantal slowing reveals dual networks for respiratory rhythm generation. Neuron 37:821-826.

Moreira TS, Takakura AC, Colombari E, Guyenet PG (2006) Central chemoreceptors and sympathetic vasomotor outflow. J Physiol 577:369-386

Moreira TS, Takakura AC, Colombari E, West GH, Guyenet PG (2007) Inhibitory input from slowly adapting lung stretch receptors to retrotrapezoid nucleus chemoreceptors. J Physiol 580:285-300.

Mulkey DK, Stornetta RL, Weston MC, Simmons JR, Parker A, Bayliss DA, Guyenet PG (2004) Respiratory control by ventral surface chemoreceptor neurons in rats. Nat Neurosci 7:1360-1369.

Nattie E, Li A (2009) Central chemoreception is a complex system function that involves multiple brain stem sites. J Appl Physiol 106:1464-1466.

Oku Y, Masumiya H, Okada Y (2007) Postnatal developmental changes in activation profiles of the respiratory neuronal network in the rat ventral medulla. J Physiol 585:175-186.

Onimaru H, Homma I (2003) A novel functional neuron group for respiratory rhythm generation in the ventral medulla. J Neurosci 23:1478-1486.

Onimaru H, Ikeda K, Kawakami K (2008) CO2-sensitive preinspiratory neurons of the parafacial respiratory group express Phox $2 \mathrm{~b}$ in the neonatal Rat. J Neurosci 28:12845-12850.

Pagliardini S, Janczewski WA, Tan W, Dickson CT, Deisseroth K, Feldman JL (2011) Active expiration induced by excitation of ventral medulla in adult anesthetized rats. J Neurosci 31:2895-2905.

Pattyn A, Morin X, Cremer H, Goridis C, Brunet JF (1997) Expression and interactions of the two closely related homeobox genes Phox $2 \mathrm{a}$ and Phox2b during neurogenesis. Development 124:4065-4075.

Paxinos G, Watson C (1986) The rat brain in stereotaxic coordinates. Sydney: Academic.

Potts JT, Rybak IA, Paton JF (2005) Respiratory rhythm entrainment by somatic afferent stimulation. J Neurosci 25:1965-1978.

Rosin DL, Chang DA, Guyenet PG (2006) Afferent and efferent connections of the rat retrotrapezoid nucleus. J Comp Neurol 499:64-89.

Rubin JE, Shevtsova NA, Ermentrout GB, Smith JC, Rybak IA (2009) Multiple rhythmic states in a model of the respiratory central pattern generator. J Neurophysiol 101:2146-2165. 
Smith JC, Morrison DE, Ellenberger HH, Otto MR, Feldman JL (1989) Brainstem projections to the major respiratory neuron populations in the medulla of the cat. J Comp Neurol 281:69-96.

Stornetta RL, Sevigny CP, Guyenet PG (2002) Vesicular glutamate transporter DNPI/VGLUT2 mRNA is present in C1 and several other groups of brainstem catecholaminergic neurons. J Comp Neurol 444:191-206.

Stornetta RL, Moreira TS, Takakura AC, Kang BJ, Chang DA, West GH, Brunet JF, Mulkey DK, Bayliss DA, Guyenet PG (2006) Expression of Phox $2 \mathrm{~b}$ by brainstem neurons involved in chemosensory integration in the adult rat. J Neurosci 26:10305-10314.

Stornetta RL, Abbott SBG, Kanbar R, Guyenet PG (2009) Photostimulation of channelrhodopsin2(ChR2)-transfected $\mathrm{C} 1$ neurons increases sympathetic nerve discharge (SND) and blood pressure (BP) in rats. Soc Neurosci Abstr 35:467.5.

Takakura AC, Moreira TS, Colombari E, West GH, Stornetta RL, Guyenet PG
(2006) Peripheral chemoreceptor inputs to retrotrapezoid nucleus (RTN) $\mathrm{CO}_{2}$-sensitive neurons in rats. J Physiol 572:503-523.

Takakura AC, Moreira TS, Stornetta RL, West GH, Gwilt JM, Guyenet PG (2008) Selective lesion of retrotrapezoid Phox2b-expressing neurons raises the apnoeic threshold in rats. J Physiol 586:2975-2991.

Takakura AC, Colombari E, Menani JV, Moreira TS (2011) Ventrolateral medulla mechanisms involved in cardiorespiratory responses to central chemoreceptor activation in rats. Am J Physiol Regul Integr Comp Physiol 300:R501-R510.

Thoby-Brisson M, Karlén M, Wu N, Charnay P, Champagnat J, Fortin G (2009) Genetic identification of an embryonic parafacial oscillator coupling to the preBotzinger complex. Nat Neurosci 12:1028-1035.

Wittmeier S, Song G, Duffin J, Poon CS (2008) Pacemakers handshake synchronization mechanism of mammalian respiratory rhythmogenesis. Proc Natl Acad Sci U S A 105:18000-18005. 